\title{
ARTICLE \\ Gephyrin-mediated formation of inhibitory postsynaptic density sheet via phase separation
}

\author{
Guanhua Bai ${ }^{1}$, Yu Wang ${ }^{1}$ and Mingjie Zhang (iD) ${ }^{1,2}$
}

\begin{abstract}
Inhibitory synapses are also known as symmetric synapses due to their lack of prominent postsynaptic densities (PSDs) under a conventional electron microscope (EM). Recent cryo-EM tomography studies indicated that inhibitory synapses also contain PSDs, albeit with a rather thin sheet-like structure. It is not known how such inhibitory PSD (iPSD) sheet might form. Here, we demonstrate that the key inhibitory synapse scaffold protein gephyrin, when in complex with either glycine or $\mathrm{GABA}_{\mathrm{A}}$ receptors, spontaneously forms highly condensed molecular assemblies via phase separation both in solution and on supported membrane bilayers. Multivalent and specific interactions between the dimeric E-domain of gephyrin and the glycine/GABA $\mathrm{A}_{\mathrm{A}}$ receptor multimer are essential for the iPSD condensate formation. Gephyrin alone does not form condensates. The linker between the G- and E-domains of gephyrin inhibits the iPSD condensate formation via autoinhibition. Phosphorylation of specific residues in the linker or binding of target proteins such as dynein light chain to the linker domain regulates gephyrin-mediated glycine/GABA $\mathrm{A}$ receptor clustering. Thus, analogous to excitatory PSDs, iPSDs are also formed by phase separation-mediated condensation of scaffold protein/ neurotransmitter receptor complexes.
\end{abstract}

Cell Research (2021) 31:312-325; https://doi.org/10.1038/s41422-020-00433-1

\section{INTRODUCTION}

Highly condensed and dynamically regulatable clustering of ion channels at specific plasma membrane sites is crucial for efficient and accurate neuronal signal transmissions. Specialized structures of presynaptic boutons and postsynaptic dendritic spine protrusions of excitatory synapses provide spatial constraints to facilitate condensed localization of molecular components including scaffold proteins and ion channels in these micron-sized compartments. $^{1-3}$ The excitatory synapse typically contains a thick and disc-shaped postsynaptic density (PSD) that is likely formed by major scaffold proteins via phase separation. ${ }^{4,5}$ Most of inhibitory synapses are formed on cell soma or dendritic shafts, thus inhibitory synapses lack obvious postsynaptic membrane protrusions. Accordingly, inhibitory synapse does not contain discshaped PSD assembly. Instead, inhibitory synapse contains a thin sheet-like structure beneath the postsynaptic membrane. ${ }^{6}$ Nonetheless, neurotransmitter receptors in inhibitory synapses are highly clustered. ${ }^{7,8}$ Compared to excitatory postsynaptic densities (ePSDs), much less is known about how the neurotransmitter receptors in inhibitory synapses are clustered and how such clusters are dynamically regulated.

As one of the most extensively studied proteins at inhibitory synapses, gephyrin is a master organizer which links transmembrane receptors with downstream signaling proteins for synaptic signal transmissions. ${ }^{9,10}$ Interestingly, gephyrin can directly interact with the cytoskeleton. ${ }^{11-13}$ Whereas in excitatory synapses, several layers of distinct scaffold proteins interface glutamate receptors and the cytoskeleton. ${ }^{3,14}$ The above difference may explain why the inhibitory synapse lacks a thick layer of postsynaptic density as in ePSD. Gephyrin was initially identified as a $93-\mathrm{kDa}$ glycine receptor (GlyR)-associated protein cofractionated with tubulin. ${ }^{11,15}$ It is highly conserved in vertebrates and contains two structured domains: an $\mathrm{N}$-terminal trimerization G-domain (GPHN-G) and a C-terminal dimerization E-domain (GPHN-E) linked by a flexible C-domain (Fig. 1a). GlyR and GABA receptors $\left(G A B A_{A} R s\right)$, via their intracellular loops, bind to the Edomain of gephyrin ${ }^{16}$ (Fig. 1a). Considering the oligomerization state of the G- and E-domains, a hexagonal gephyrin lattice model has been proposed ${ }^{17}$ and regarded as a popular framework for gephyrin-mediated molecular organizations in inhibitory synapses. ${ }^{9,10}$ However, the hexagonal lattice structure model has not been observed for gephyrin in vitro or in synapses. It is possible that the flexible C-domain prevents gephyrin from forming the highly regular lattice structure. ${ }^{18}$ In addition to functioning as a linker of the two structured domains, the Cdomain is known to regulate gephyrin function by binding to target proteins or through post-translational modifications., 10,19 Loss of gephyrin causes mice to die within 1 day after birth. In neurons of gephyrin-deficient mice, synaptic clustering of both GlyRs and $\mathrm{GABA}_{A} \mathrm{Rs}$ are markedly reduced, ${ }^{20}$ indicating the essential role of gephyrin in receptor accumulation at inhibitory synapses. A number of gephyrin mutations have been associated with neuronal disorders including autism, schizophrenia, and epilepsy. ${ }^{21-23}$ However, the molecular basis underlying gephyrinmediated GlyRs and $\mathrm{GABA}_{A} \mathrm{Rs}$ organization and clustering remain largely unclear.

Cells are highly compartmentalized. In addition to classical membrane-enclosed cellular compartments, increasing evidence in recent years is revealing a diverse class of cellular compartments that either lack or are not enclosed by membranes. These

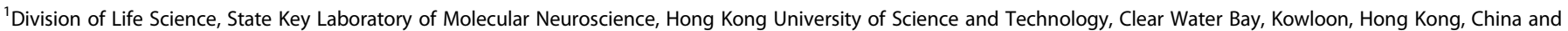
${ }^{2}$ Center of Systems Biology and Human Health, Hong Kong University of Science and Technology, Clear Water Bay, Kowloon, Hong Kong, China
}

Correspondence: Mingjie Zhang (mzhang@ust.hk)

Received: 27 June 2020 Accepted: 14 October 2020

Published online: 2 November 2020 

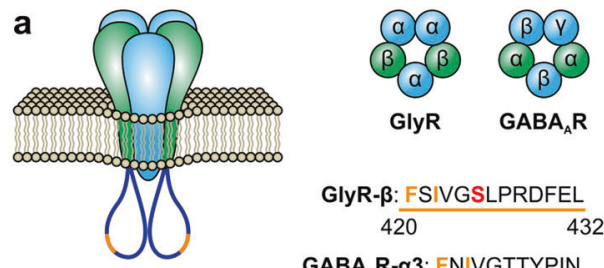

GlyR- $\beta$ : FSIVGSLPRDFEL

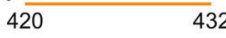

GABA R- $\alpha 3$ : FNIVGTTYPIN
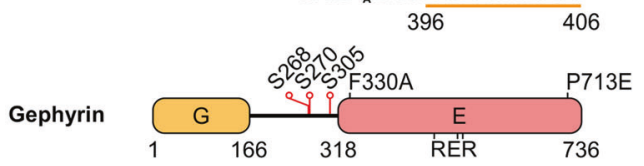

C
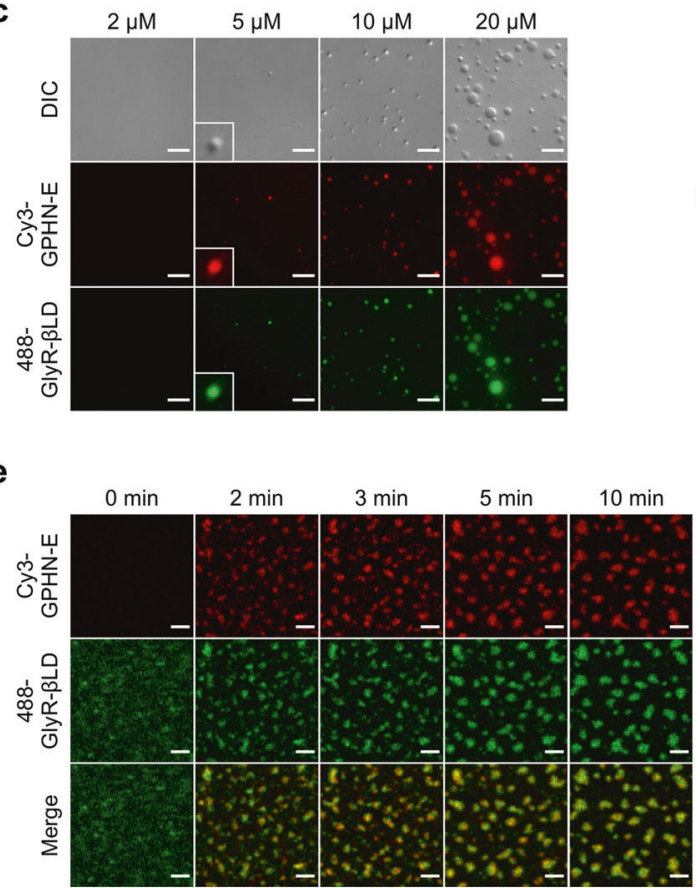

b GPHN-E

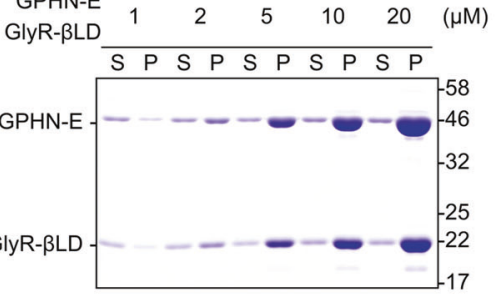

GPHN-E GlyR-ßLD
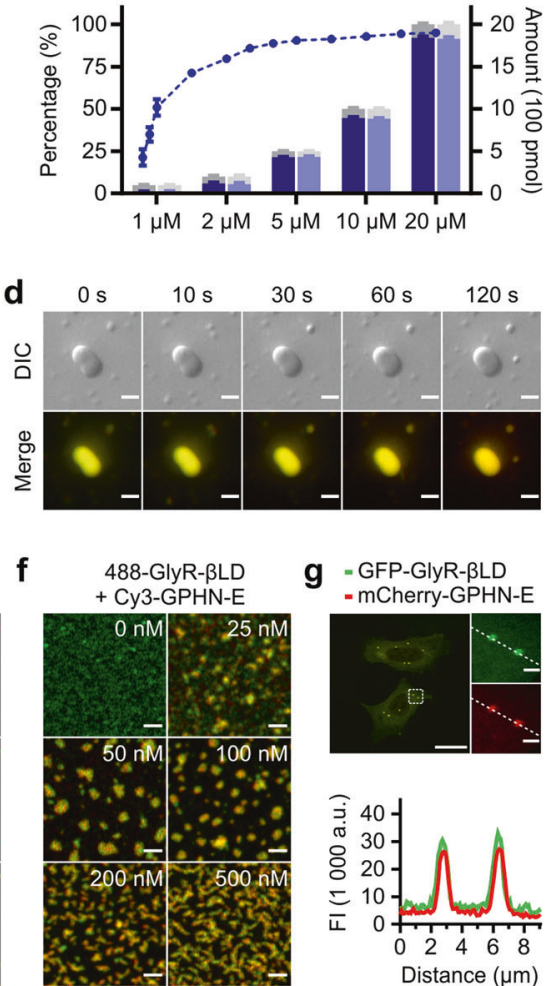

g = GFP-GlyR- $\beta$ LD - mCherry-GPHN-E
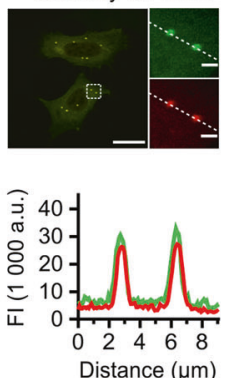

Fig. 1 Phase separation of the GPHN-E/GlyR- $\beta$ LD complex. a Schematic diagrams showing the pentameric subunit assembly of GlyR or $G_{A B A} R$. The gephyrin binding core sequences in the cytoplasmic TM3-4 loops of the receptors are highlighted by an orange line and the amino acid sequences are shown. The figure also shows the domain organization of gephyrin. The positions of mutations introduced into the recombinant receptor loops and gephyrin proteins, as well as three potential phosphorylation sites in the gephyrin C-domain are indicated. b Representative SDS-PAGE of sedimentation experiments (upper panel) and quantification of relative (line graph, left y-axis) and absolute (bar graph, right $y$-axis) amount of proteins recovered in the supernatant ( $S$, gray columns) and pellet ( $P$, blue columns) (lower panel) in the assay. Proteins were mixed at the indicated concentrations. Data from three different batches of experiments were presented as means \pm SD. $c$ DIC and fluorescence images showing that mixtures of Cy3-GPHN-E and 488-GlyR- $\beta \mathrm{LD}$ at the 1:1 molar ratio and indicated concentrations formed phase-separated droplets. The boxes show a $5 \times$ zoom-in analysis of a droplet at the $5 \mu \mathrm{M}$ of the protein concentration. Scale bars, $10 \mu \mathrm{m}$. $\mathbf{d}$ Time-lapse imaging showing the fusion of two small droplets into a larger one. The concentration of the protein mixture was $20 \mu \mathrm{M}$. Scale bars, $5 \mu \mathrm{m}$. e Time-lapse imaging showing that membrane-tethered 488-GlyR- $\beta$ LD gradually formed clusters on lipid bilayers upon the addition of $100 \mathrm{nM}$ Cy3-GPHN-E. Scale bars, $2 \mu \mathrm{m}$. f Fluorescence images showing that the clustering patterns of membrane-tethered 488GlyR- $\beta$ LD on lipid bilayers depended on the concentration of Cy3-GPHN-E. Cy3-GPHN-E was added at the indicated concentrations. Scale bars, $2 \mu \mathrm{m}$. g Top panel: representative fluorescence images showing that co-expression of mCherry-GPHN-E with GFP-GlyR- $\beta$ LD in HeLa cells led to the formation of many puncta with the two proteins colocalized together in each punctum. These puncta are not enriched with membranes (Fig. S4a). The dashed box is magnified and shown at right. Scale bars, $20 \mu \mathrm{m}$. Scale bars for the zoomed-in images are $2 \mu \mathrm{m}$. Bottom panel: fluorescence intensity line-scanning plots showing that both proteins were concentrated and colocalized together in the two bright puncta shown in the zoomed-in images.

compartments can autonomously form via liquid-liquid phase separation and are frequently referred to as membraneless compartments or biological condensates. Formation of membraneless compartments is increasingly recognized as a general strategy for diverse cellular processes including cell polarity establishment and maintenance, cell signaling, cell and organ development, cell survival, and aging. ${ }^{24-26}$ Neurons take cellular compartmentalization to the extreme due to their elaborate morphologies and a high degree of polarity. Formation of membraneless compartments by phase separation is especially important both for neuronal development and for proper functions in adult neurons. ${ }^{27}$

In this study, we demonstrate that gephyrin, upon binding to GlyR or $\mathrm{GABA}_{A} \mathrm{R}$, can autonomously form highly condensed and dynamic assemblies via phase separation. The phase separation of the gephyrin/receptor complex is driven by highly specific and multivalent interactions between the two proteins. Importantly, the C-domain inhibits the phase separation capacity of gephyrin, and this autoinhibition is regulated by post-translational modifications. Our study, together with a recent cryo-electron tomography 
study of inhibitory synapses in situ, ${ }^{28}$ provide compelling evidence showing that inhibitory synapses contain thin-sheet like PSDs primarily formed by gephyrin via receptor binding-induced phase separation.

\section{RESULTS}

Binding of dimerized GlyR $\beta$ subunit cytoplasmic loop triggers phase separation of GPHN-E

GlyRs, encoded by four a subunits (a1-a4) and one $\beta$ subunit, are abundantly expressed in spinal cords, brain stems, and in different brain regions. ${ }^{29}$ GlyRs in the spinal cord of adult mammals are heteropentamers generally composed of three $a$ and two $\beta$ subunits ${ }^{29,30}$ (Fig. 1a). The a subunits harbor critical determinants for ligand binding and allosteric modulation and thus control GlyRs' function. The $\beta$ subunit alone cannot form GlyRs. The two $\beta$ subunits in GlyRs mainly play structural roles as well as mediate the intracellular trafficking and postsynaptic clustering of the receptor through binding to gephyrin. ${ }^{31,32}$ The large cytoplasmic TM3-4 loop of GlyR $\beta$ (GlyR- $\beta$ L) interacts with GPHN-E. ${ }^{33}$ The crystal structure of GPHN-E/GlyR- $\beta$ L (aa 400-448) complex reveals that each GPHN-E dimer binds to two GlyR- $\beta$ L molecules. A 13residue core sequence of GlyR- $\beta$ L (aa 420-432) is responsible for binding to GPHN-E ${ }^{34,35}$ (Fig. 1a). A recent study indicated that residues flanking the 13-residue core also contribute to GlyR synaptic trapping by gephyrin. ${ }^{36}$

We purified the entire GlyR- $\beta$ L (aa 349-474) fused to the tail of a dimeric GCN4 coiled-coil domain (referred to as GlyR- $\beta$ LD) to partially mimic the dimeric nature of the $\beta$ subunits in the heteropentameric GlyRs (3a:2 $\beta$; Fig. 1a). GlyR- $\beta$ LD (aa 349-474) exhibited a similar binding affinity in binding to GPHN-E compared with GlyR- $\beta$ LD (aa 400-448) (Supplementary information, Fig. S1a, b). Careful inspection of the isothermal titration calorimetry (ITC) traces revealed that the longer form of GlyR- $\beta$ LD showed an obvious dip when the molar ratio of GlyR- $\beta$ LD to GPHN-E was in 0.5-1.0, which was caused by additional heat releases due to phase separation of the complex (Fig. 2d, e; Supplementary information, Fig. S1a, b). Mixing purified GlyR- $\beta$ LD and GPHN-E at low $\mu \mathrm{M}$ concentrations led the solutions to become opalescent. Using a sedimentation-based assay, we separated and quantified the amount of proteins in the condensed and the bulk dilute phases. The percentage of the GPHN-E/GlyR- $\beta$ LD complex recovered in the pellets (i.e., the condensed phase) reached the saturation level when the concentration of each protein was at $\sim 5 \mu \mathrm{M}$ or above (Fig. 1b). Differential interference contrast (DIC) and fluorescence images showed that the GPHN-E/GlyR- $\beta$ LD mixtures at the concentrations $\sim 5 \mu \mathrm{M}$ or above readily formed spherical droplets with both proteins highly enriched within (Fig. 1c). At lower concentrations such as $2 \mu \mathrm{M}$ in Fig. $1 \mathrm{c}$, the GPHN-E/GlyR- $\beta$ LD mixtures formed very small droplets and these droplets did not immediately settle to the surface of the glass slide. We had to wait for a long time for very fine droplets to coarsen and settle on the slide surface for imaging. Upon contact with each other, small droplets fused with each other forming larger droplets over time (Fig. 1d). Neither GlyR- $\beta$ LD nor GPHN-E alone at concentrations up to $500 \mu \mathrm{M}$ tested showed any sign of phase separation. Fluorescence recovery after photobleaching (FRAP) experiments showed that both components could dynamically diffuse between the condensed phase and the dilute phase (Supplementary information, Fig. S2a, b). Thus, we conclude that the formation of the GPHN-E/GlyR- $\beta$ LD complex triggers phase separation-mediated condensation of both proteins.

Since gephyrin clusters GlyRs on the postsynaptic membranes at inhibitory synapses, ${ }^{20,37}$ we next tested whether GPHN-E could induce clustering of GlyR- $\beta$ LD tethered to supported lipid bilayers (Supplementary information, Fig. S3). Upon the addition of GPHN$E$, we observed submicron-sized GlyR- $\beta$ LD cluster formation and time-dependent cluster coarsening (Fig. 1e), indicating that the GPHN-E/GlyR- $\beta$ LD complex formed condensed clusters via phase separation. The threshold concentration required for the GPHN-E/ GlyR- $\beta$ LD complex to undergo phase separation on lipid bilayers was as low as $25 \mathrm{nM}$ (Fig. 1f). When the concentration of the GPHN-E/GlyR- $\beta$ LD complex on lipid bilayer gradually increased, the phase separation changed from binodal nucleation to spinodal decomposition, a process also observed for the phase separation of ePSD condensates on lipid bilayers. ${ }^{5}$ FRAP assays also showed that GPHN-E could exchange between the condensed phase and the dilute phase on lipid bilayers (Supplementary information, Fig. S2c, d).

We also co-expressed mCherry-tagged GPHN-E (mCherryGPHN-E) with GFP-tagged GlyR- $\beta$ LD (GFP-GlyR- $\beta$ LD) in HeLa cells and observed many bright membraneless puncta with both proteins perfectly co-localized (Fig. 1g; Supplementary information, Fig. S4a). No puncta formed in cells when only mCherryGPHN-E or GFP-GlyR- $\beta$ LD was expressed (Supplementary information, Fig. S4b). Taken together, the results in Fig. 1 demonstrate that binding of GlyR- $\beta$ LD to GPHN-E triggers the GPHN-E/GlyR- $\beta$ LD complex to form condensed clusters in solution and on membranes via phase separation.

Multivalent interactions between GlyR- $\beta$ L and GPHN-E are required for the phase separation

We next used sedimentation assays to evaluate the impact of various mutations of GlyR- $\beta$ LD and GPHN-E on the condensate formation of the complex. Compared with the wild-type (WT) proteins, mutations (F420A/I422A on GlyR- $\beta L D$, termed as " $\beta F I^{\prime}$; F330A or P713E on GPHN-E) reducing or abolishing the binding between GlyR- $\beta$ LD and $G P H N-E^{35}$ prevented the condensate formation (Fig. 2a; Supplementary information, Fig. S1e-g), indicating that the direct binding is required for the phase separation of the GPHN-E/GlyR- $\beta$ LD complex to occur.

Phosphorylation of $\mathrm{S} 425$ within the GlyR- $\beta \mathrm{L}$ core binding sequence has been reported to perturb its binding to GPHN-E and to cause reduced GlyR synaptic clustering. ${ }^{38}$ Surprisingly, compared to the WT protein, the affinity of the GlyR- $\beta$ LD_S425D mutant in binding to GPHN-E was only slightly reduced $\left(K_{\mathrm{D}}\right.$ values of $230 \mathrm{nM}$ for WT vs. $389 \mathrm{nM}$ for the mutant; Supplementary information, Fig. S1b, h), but the mutant lost the phase separation capacity when in complex with GPHN-E (Fig. 2a). Correspondingly, the ITC curve of GlyR- $\beta$ LD_S425D binding to GPHN-E was smooth (i.e., lacking a dip corresponding to the phase separation of GlyR$\beta L D \_W T$ binding to GPHN-E) (Supplementary information, Fig. S1b, h). This finding suggests that the phase separation of the GPHN-E/ GlyR- $\beta$ LD complex is highly sensitive to the charge properties of the complex, phosphorylation of S425 can modulate gephyrinmediated synaptic clustering of GlyR without obviously altering their binding.

Next, we assessed the role of GlyR- $\beta$ L and GPHN-E oligomerization in the phase separation of the complex. ${ }^{39}$ The monomeric GlyR- $\beta$ L (GlyR- $\beta L M$ ) retained the high-affinity binding to GPHN-E (i.e., GlyR- $\beta$ LM and GlyR- $\beta$ LD display similar binding affinity to GPHN-E; Supplementary information, Fig. S1C, i). However, GlyR$\beta L M$ could hardly trigger phase separation after mixing with GPHN-E (Fig. 2b), suggesting that the presence of multiple $\beta$ subunits in GlyR is critical for gephyrin-mediated receptor clustering. Consistently, co-expression of GFP-tagged monomeric GlyR- $\beta$ LM with GPHN-E resulted in a much lower number of the GlyR- $\beta$ LM/GPHN-E puncta and dramatically reduced enrichment of GlyR- $\beta$ LM in the puncta in HeLa cells (Supplementary information, Fig. S4c). Since some evidence are suggesting that each GlyR may contain three $\beta$ subunits, ${ }^{40,41}$ we also examined a trimerized GlyR$\beta L$ (GlyR- $\beta$ LT; Supplementary information, Fig. S1j) in inducing phase separation of GPHN-E. The sedimentation assay showed that the dimeric and trimeric versions of GlyR had no differences in inducing phase separation of GPHN-E (Fig. 2b), presumably 
a
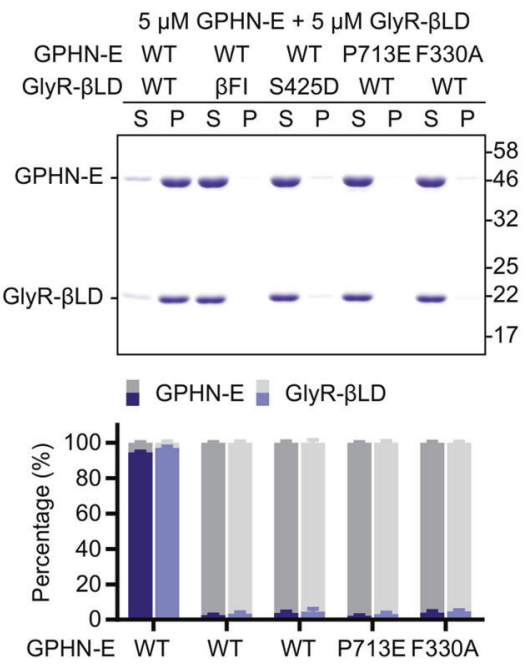

GlyR- $\beta$ LD WT $\quad$ WFI S425D WT WT b $5 \mu \mathrm{M}$ GPHN-E + $5 \mu \mathrm{M}$ GlyR- $\beta$ LM/D/T GPHN-E WT WT WT RER

GlyR- $\beta$ LM $\beta L D \quad \beta L T \quad \beta L D$
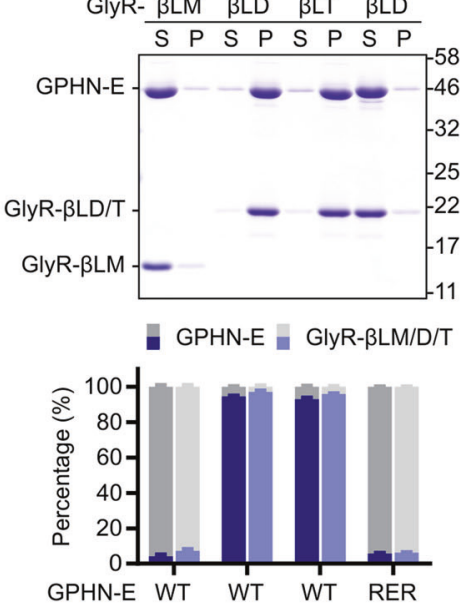

GlyR- $\beta L M \quad \beta L D \quad \beta L T \quad \beta L D$

C Cy3-GPHN-E $20 \mu \mathrm{M} \quad 15 \mu \mathrm{M} \quad 10 \mu \mathrm{M}$ 488-GlyR-ßLD $5 \mu \mathrm{M} \quad 5 \mu \mathrm{M} \quad 5 \mu \mathrm{M}$

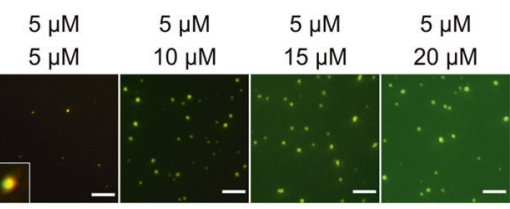

d

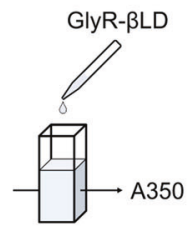

GPHN-E
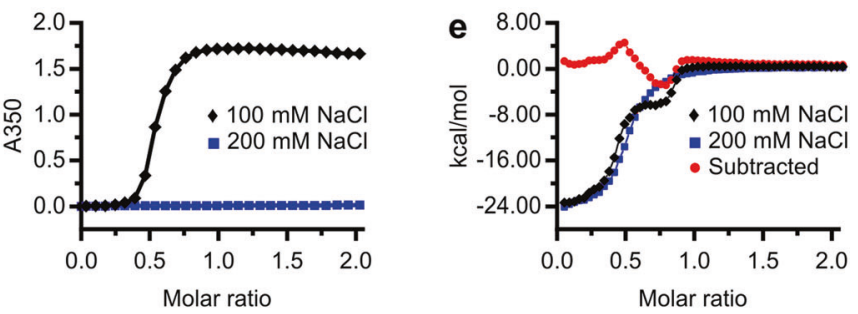

f
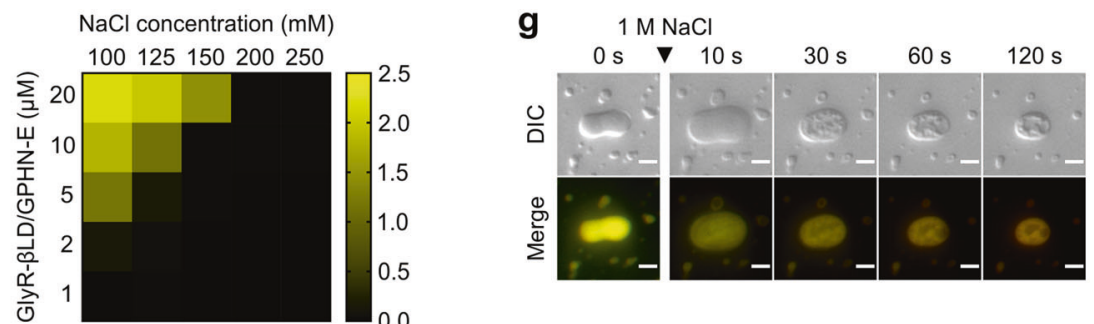

Fig. 2 Multivalent interactions between GlyR- $\beta$ L and GPHN-E are required for the phase separation of the complex. a Representative SDSPAGE of sedimentation experiments (upper panel) and quantification of protein distributions in the supernatant (S, gray columns) and pellet ( $\mathrm{P}$, blue columns) (lower panel) showing the phase separation capacities of different mutant proteins. Data from three different batches of experiments were presented as means \pm SD. b Representative SDS-PAGE of sedimentation experiments (upper panel) and quantification of protein distributions in the supernatant ( $\mathrm{S}$, gray columns) and pellet ( $\mathrm{P}$, blue columns) (lower panel) showing the phase separation capacities of proteins with different oligomerization states. Data from three different batches of experiments were presented as means \pm SD. c Fluorescence images showing the phase separation of the Cy3-GPHN-E/488-GlyR- $\beta$ LD mixtures at the indicated protein concentrations/ratios. Scale bars, $10 \mu \mathrm{m}$. d Turbidity assay of the phase separation of the GPHN-E/GlyR- $\beta$ LD complex. GlyR- $\beta$ LD (100 $\mu \mathrm{M})$ was titrated into a $10 \mu \mathrm{M}$ GPHN-E solution in a $1 \mathrm{~cm}$ cuvette with $100 \mathrm{mM} \mathrm{NaCl}$ (black) or $200 \mathrm{mM} \mathrm{NaCl}$ (blue) in the assay buffer solution, the absorbance at $350 \mathrm{~nm}$ at each titration point was measured. e Representative high-resolution ITC titration isotherms showing the titrations of $100 \mu \mathrm{M}$ GlyR- $\beta$ LD into the reaction cell containing $10 \mu \mathrm{M}$ GPHN-E with $100 \mathrm{mM} \mathrm{NaCl}$ (black) or with $200 \mathrm{mM} \mathrm{NaCl}$ (blue) in the binding buffer. The red curve is derived by subtracting the blue isotherms from the black isotherms and represents the enthalpy changes resulted from the phase separation of the GPHN-E/GlyR- $\beta$ LD complex during the titration. $f$ Phase diagram showing the phase separation of the GPHN-E/GlyR- $\beta$ LD complex as functions of buffer $\mathrm{NaCl}$ concentration and protein concentrations. The degree of phase separation at each data point was derived from the turbidity assay as described in d. g Time-lapse DIC and fluorescence imaging showing the dispersion of the GPHN-E/GlyR- $\beta$ LD complex droplets by the addition of $\mathrm{NaCl}$ to the sample buffer. Proteins were initially mixed at $20 \mu \mathrm{M}$ at the 1:1 ratio. Scale bars, $5 \mu \mathrm{m}$. 
because that each GPHN-E dimer contains only two specific binding sites for the GlyR $\beta$ subunit. A triple mutation of GPHN-E (G483R/R523E/A532R, termed as "GPHN-E_RER"), which is known to disrupt its dimer formation ${ }^{42}$ and also largely decreases its binding to GlyR- $\beta$ L (Supplementary information, Fig. S1k), could not phase separate with GlyR- $\beta$ LD (Fig. 2b).

We further found that the phase separation of the GPHN-E/ GlyR- $\beta$ LD mixture was sensitive to the molar ratio of two proteins. Starting at $5 \mu \mathrm{M}$ for both proteins, increasing the amount of GlyR$\beta L D$ only slightly increased the phase separation of the complex, and the system quickly reached saturation. Conversely, the addition of excess amounts of GPHN-E gradually dispersed the condensates formed by the GPHN-E/GlyR- $\beta$ LD complex (Fig. 2c; Supplementary information, Fig. S5a, b), presumably because when GPHN-E is in excess, each GPHN-E dimer no longer always binds to the two peptide fragments from the same GlyR$\beta L D$ dimer.

We adopted a turbidity-based assay to specifically monitor the phase separation of the GPHN-E/GlyR- $\beta$ LD complex. We titrated GlyR- $\beta$ LD into a GPHN-E solution $(10 \mu \mathrm{M}$ in buffer containing 100 $\mathrm{mM} \mathrm{NaCl}$ ), and monitored the light scattering by A350. Obvious phase separation was observed when the concentration of GlyR$\beta L D$ reached $5 \mu \mathrm{M}$ (i.e., the GlyR- $\beta$ LD to GPHN-E ratio at $0.5: 1$ ) and phase separation became saturated when the GlyR- $\beta$ LD to GPHN$E$ ratio reached $\sim 1: 1$ (Fig. $2 d$, black curve). Taken together, the results presented above demonstrated that the formation of the GPHN-E/GlyR- $\beta$ LD condensates requires specific and multivalent interactions between the two proteins. Additionally, due to the highly specific interaction between the two proteins, the phase separation of the complex is also sensitive to the relative ratio of the two proteins.

Phase separation of the GPHN-E/GlyR- $\beta$ LD complex is highly sensitive to salt concentrations

The binding between GlyR- $\beta$ L and GPHN-E is mainly mediated by hydrophobic interactions. ${ }^{35,43}$ Consistent with structural analysis, increasing $\mathrm{NaCl}$ concentration in the binding buffer from $100 \mathrm{mM}$ to $200 \mathrm{mM}$ did not weaken the interaction between GlyR- $\beta \mathrm{L}$ and GPHN-E $\left(K_{\mathrm{D}}\right.$ values of $230 \mathrm{nM}$ at $100 \mathrm{mM} \mathrm{NaCl}$ vs. $181 \mathrm{nM}$ at 200 $\mathrm{mM} \mathrm{NaCl}$; Supplementary information, Fig. S1b, I). Strikingly, the GPHN-E/GlyR- $\beta$ LD complex was incapable of undergoing phase separation when the buffer $\mathrm{NaCl}$ concentration was raised to 200 mM (Fig. 2d, e; Supplementary information, Fig. S5c, d). By subtracting the ITC curve of the titration in $100 \mathrm{mM} \mathrm{NaCl}$ with that in $200 \mathrm{mM} \mathrm{NaCl}$, we obtained a concentration-dependent enthalpy change profile for the phase separation of the GPHN-E/ GlyR- $\beta$ LD complex (the red curve in Fig. 2e; original curves in Supplementary information, Fig. S1c, d). The result showed that the formation of the GPHN-E/GlyR- $\beta$ LD condensates is an enthalpy-driven process. We further constructed a phase diagram showing the protein concentration- and buffer salt concentrationdependent phase separation of the GPHN-E/GlyR- $\beta$ LD complex (Fig. 2f). The result showed that the phase separation of the GPHNE/GlyR- $\beta$ LD complex is very sensitive to the buffer salt concentration. Time-lapse fluorescent images also showed that GPHN-E/ GlyR- $\beta$ LD droplets can be readily dispersed by raising salt concentration in the mixture (Fig. $2 \mathrm{~g}$ ). The hypersensitivity of the GPHN-E/GlyR- $\beta$ LD complex phase separation to salt concentration suggests that the clustering of GlyR by gephyrin may be readily regulated by factors such as activity-induced ion influx in synapse and/or phosphorylation of either/both proteins.

Molecular determinants of GlyR- $\beta$ L governing its phase separation with GPHN-E

Though sharing a similar binding affinity with GlyR- $\beta$ LD (aa 349-474) to GPHN-E, GlyR- $\beta$ LD (aa 400-448, defined as "Core" in Fig. 3a) did not undergo phase separation with GPHN-E (Supplementary information, Figs. S1a, b, and S6a), suggesting that amino acids outside of this core sequence play essential roles in the phase separation of the GPHN-E/GlyR- $\beta$ LD complex. Sequence analysis of GlyR- $\beta$ L revealed that, although unstructured, the sequence of the entire loop is extremely conserved among vertebrates. The fragments flanking both ends of the core are enriched with charged residues (Fig. 3a). Deletion analysis showed that removal of the C-terminal fragment (" $\left.\Delta C^{\prime \prime}\right)$ did not affect, but the removal of the N-terminal fragment $\left(" \Delta N^{\prime \prime}\right)$ abolished the phase separation of the GPHN-E/GlyR- $\beta$ LD complex (Fig. 3a; Supplementary information, Fig. S6a). We substituted some of the charged residues in part of the $\mathrm{N}$-terminal fragment (aa 354-383; Fig. 3a) with serine ("+ve6S", short for substituting 6 Arg and Lys residues with Ser; "-ve4S", short for replacing 4 Glu and Asp with Ser; "+/-veS", short for replacing all 10 charged residues with Ser). Both imaging and sedimentation assays showed that removing positive charges (i.e., "+ve6S") dramatically reduced phase separation, removing negatively charged residues (i.e., "-ve4S") promoted phase separation, and replacing all 10 charged residues with Ser (i.e., " $+/-$ veS") had a negligible impact on the phase separation of the complex (Fig. 3b, c; Supplementary information, Fig. S6a). We further showed that the reduced phase separation of the GlyR- $\beta$ LD_"+ve6S" mutant with GPHN-E was not because of the interruption of the binding introduced by the mutation (Supplementary information, Fig. S6b). The above results, combined with the salt concentration-dependent formation of the GPHN-E/GlyR-BLD condensates (Fig. 2d-f), indicated that charge-charge interactions are important for the phase separation of the GPHN-E/GlyR- $\beta$ LD complex.

A negatively charged surface away from the GlyR- $\beta$ L core binding site on GPHN-E participates in binding to GlyR- $\beta$ L and is critical for the phase separation

The GPHN-E dimer contains a highly negatively charged surface in its subdomain II (highlighted with a dashed circle in Fig. 3d). We substituted 6 negatively charged residues clustered on the surface of this subdomain with Ser ("ED6S"). The "ED6S" mutation had a negligible impact on GPHN-E's binding to GlyR- $\beta$ LD (Supplementary information, Fig. S6c). Strikingly, the "ED6S" mutation greatly reduced the phase separation capacity of the GPHN-E/GlyR- $\beta$ LD complex (Fig. 3e-g). Interestingly, a missense mutation (p.G375D) of GPHN identified in a patient with Dravet-like syndrome is in the subdomain II. ${ }^{44}$ Though the G375D mutation does not alter the binding of GPHN-E to GlyR- $\beta$ LD (Supplementary information, Fig. S6d), the GPHN-E_G375D mutant lost the ability to form condensates with GlyR- $\beta$ LD (Fig. $3 g$ ). Thus, we conclude that a second binding site formed between the positively charged residue $\mathrm{N}$-terminal to the core sequence of GlyR- $\beta \mathrm{L}$ and the negatively charged subdomain II of GPHN-E is critical for the phase separation of the GPHN-E/GlyR-BLD complex. This second binding site may allow one GlyR- $\beta$ L to cross-link two molecules of the GPHN-E dimer and thus promote the phase separation of the GPHN-E/GlyR- $\beta$ LD complex.

\section{Gephyrin C-domain inhibits its E-domain-mediated phase} separation with GlyR- $\beta$ LD

Full-length gephyrin expressed in heterologous cells forms large intracellular puncta in $\sim 20 \%$ of cells (Fig. $4 a, b){ }^{33,42}$ Co-expression of GFP-tagged monomeric or dimeric GlyR- $\beta$ L with mCherrytagged full-length gephyrin (mCherry-GPHN) led to the formation of puncta in nearly all cells with the two proteins perfectly colocalized and a dramatic increase in the number of puncta in each cell (Fig. 4a, b). A lipophilic dye staining experiment showed that these puncta are not membrane demarcated (Supplementary information, Fig. S7a). Additionally, FRAP assay showed that both GPHN and GlyR- $\beta$ L in the puncta could exchange with their counterparts in dilute cytoplasm (Supplementary information, Fig. S7b, c). Thus, the GPHN/GlyR- $\beta$ L puncta observed in Hela cells are likely membraneless condensates. 
a

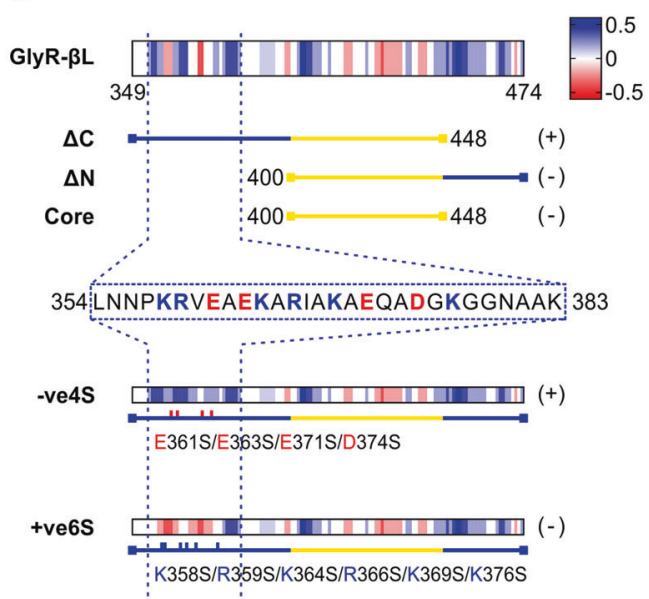

b

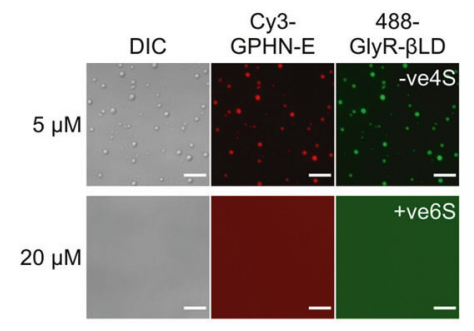

C

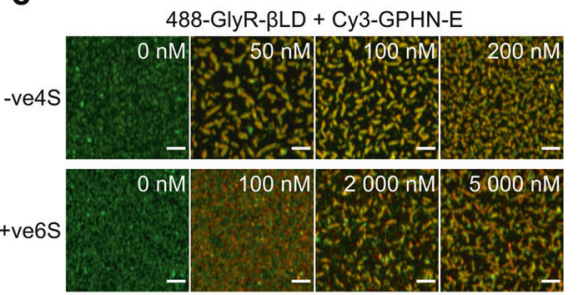

d

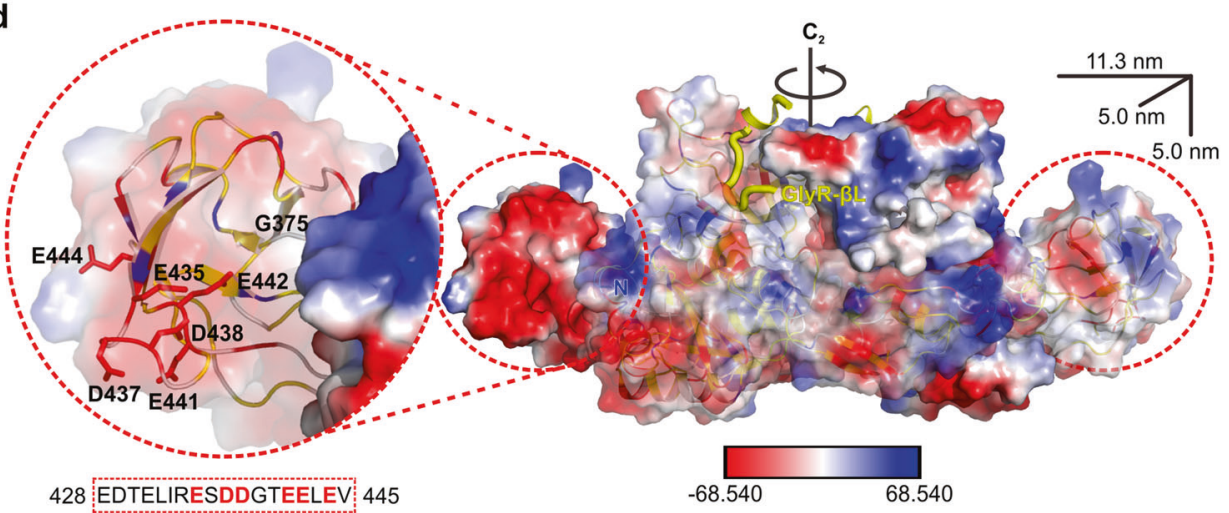

e

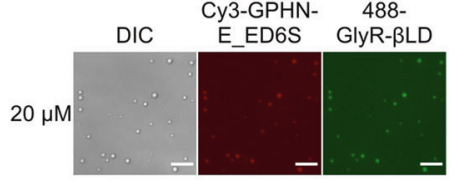

f

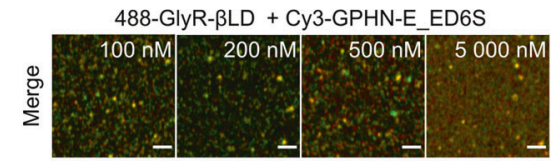

g

- GPHN-E_WT/ED6S/G375D

- GlyR-BLD

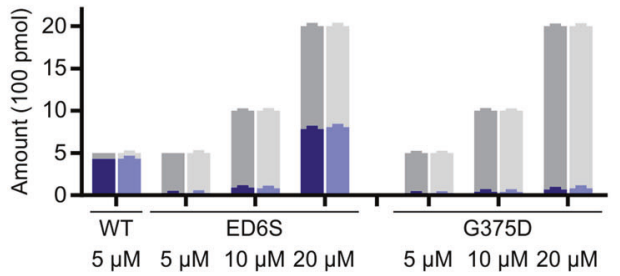

Fig. 3 Charge-charge interactions are critical for the phase separation of the GPHN-E/GlyR- $\beta$ LD complex. a Heat maps showing the net charge distributions of the TM3-4 loop of the GlyR $\beta$ subunit. The schematic diagrams also show the deletion or mutation constructs used in the study. In the diagram, "-ve4S" is short for replacing 4 Glu and Asp with Ser; "+ve6S" stands for substituting 6 Arg and Lys residues with Ser. Mutations of GlyR- $\beta$ L that led to failed phase separation with GPHN-E are indicated by $(-)$, and the mutations that retained the ability to phase separate with GPHN-E are indicated by (+). See also Supplementary information, Fig. S6a. b DIC and fluorescence images showing the enhanced phase separation of the Cy3-GPHN-E/488-GlyR- $\beta L_{-}$"-ve4S" complex at $5 \mu \mathrm{M}$ and abolished phase separation of the Cy3-GPHN-E/ 488-GlyR- $\beta$ LD_"+ve6S" complex at $20 \mu \mathrm{M}$ compared to the WT' protein complex in solution (Fig. 1c). Scale bars, $10 \mu \mathrm{m}$. c Fluorescence images

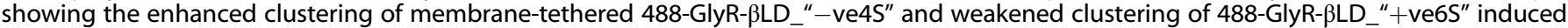
by Cy3-GPHN-E on lipid bilayers (compared with Fig. 1f). Cy3-GPHN-E was added at the indicated concentrations. Scale bars, $2 \mu \mathrm{m}$. $\mathbf{d}$ Surface charge potential map of the GPHN-E dimer (PDB: 5ERQ) in complex with GlyR- $\beta$ L (shown in the yellow ribbon and tube model). The highly negatively charged surface of the GPHN-E subdomain II is highlighted with a red circle and enlarged to show the clustering of 6 negatively charged residues and the position of G375 in the domain. The corresponding amino acid sequences are shown at the bottom. The physical dimensions of GPHN-E dimer are indicated at the upper right corner. e DIC and fluorescence images showing the weakened phase separation of the Cy3-GPHN-E_ED6S/488-GlyR- $\beta$ LD complex at $20 \mu \mathrm{M}$ compared to the WT protein complex (Fig. $1 \mathrm{c}$ ). Scale bars, $10 \mu \mathrm{m}$. $\mathbf{f}$ Fluorescence images showing that membrane-tethered 488-GlyR- $\beta$ LD could not be clustered by Cy3-GPHN-E_ED6S on lipid bilayers. Cy3-GPHN-E_ED6S was added at the indicated concentrations. Scale bars, $2 \mu \mathrm{m}$. g Quantification of protein amounts recovered in the supernatant (S, gray columns) and pellet (P, blue columns) showing that GPHN-E_ED6S and GPHN-E_G375D showed decreased capacities in forming condensates with GlyR$\beta \mathrm{LD}$. GPHN-E or its mutant was mixed with GlyR- $\beta$ LD at the indicated concentrations. Data from three different batches of experiments were presented as means \pm SD. 
a
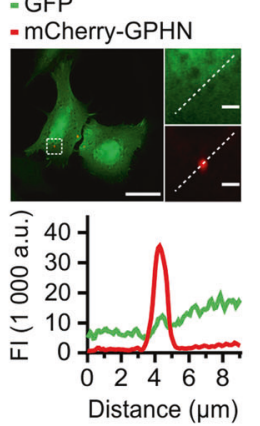
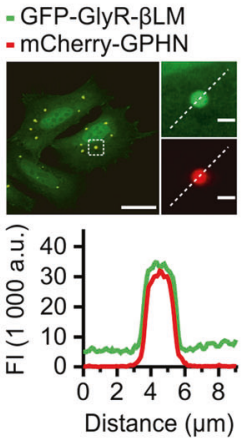
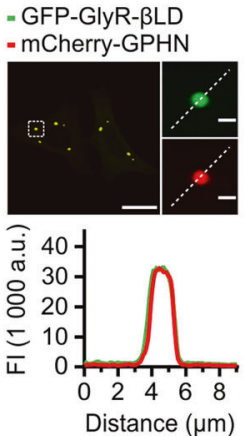

b

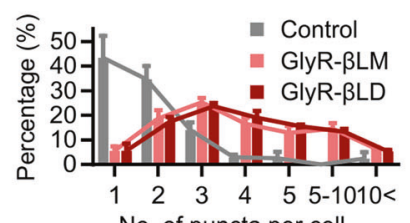

No. of puncta per cell

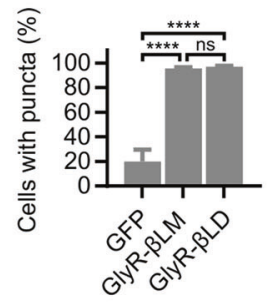

e

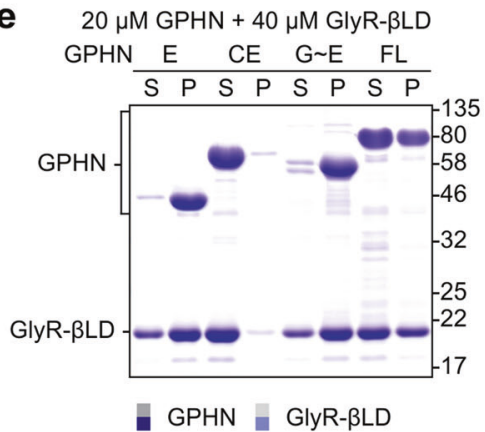

d
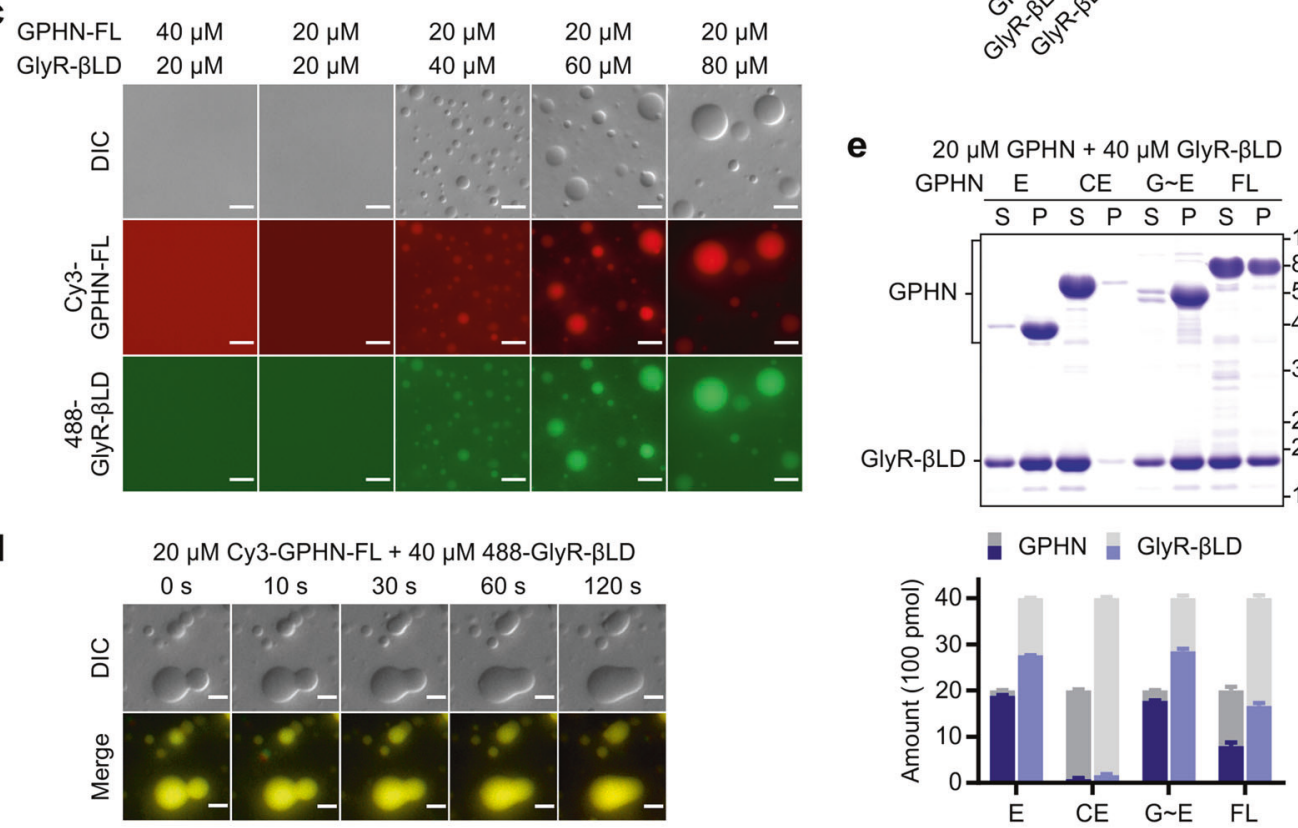

f GPHN

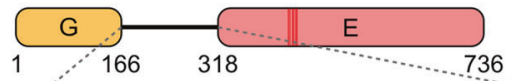

GPHN-CE

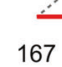

222

275

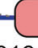

CI: DLLRDAIVKVKEVHDELEDLPSPPPPLSPPPTTSPHKQTEDKGVQCEEEEEEKKD

(DLC1 BR2) $\rightarrow($ Inhibitory Region 1)-

CII: SGVASTEDSSSSHITAAALAAKIPDSIISRGVQVLPRDTASLSTTPSESPRAQ

259

Fig. 4 The C-domain inhibits the E-domain-mediated phase separation of gephyrin with GlyR- $\boldsymbol{\beta L D}$. a Top: representative fluorescence images showing the co-expression of GFP-tagged monomeric or dimeric GlyR- $\beta$ L with gephyrin in HeLa cells. The region shown with the dashed box in each image is magnified and shown at right. Scale bars, $20 \mu \mathrm{m}$. Scale bars for the zoomed-in images, $2 \mu \mathrm{m}$. Bottom: fluorescence intensity line-scanning plots of the dashed lines in the magnified images showing that an increase of the GlyR- $\beta \mathrm{L}$ valence led to enhanced co-clustering of GFP-GlyR- $\beta$ L with mCherry-GPHN. b Quantification of the number of puncta with two proteins colocalized in puncta-positive cells and the percentage of cells containing such puncta over the total co-transfected cells. Data from three batches of cultures were presented as means \pm SD. ns, not significant; ${ }^{* * * * P}<0.0001$. c DIC and fluorescence images showing the phase separation of the Cy3-GPHN-FL/488-GlyR- $\beta$ LD mixture at different molar ratios. Proteins were mixed at indicated concentrations in $50 \mathrm{mM} \mathrm{NaCl}$ solution. Scale bars, $10 \mu \mathrm{m}$. d Time-lapse imaging showing the fusion of small droplets of the Cy3-GPHN-FL/488-GlyR- $\beta$ LD complex into larger ones. Proteins were mixed at indicated concentrations in $50 \mathrm{mM} \mathrm{NaCl}$ solution. Scale bars, $5 \mu \mathrm{m}$. e Representative SDS-PAGE of sedimentation experiments (upper panel) and quantification of protein amounts in the supernatant ( $S$, gray columns) and pellet (P, blue columns) (lower panel) showing that the gephyrin C-domain plays an inhibitory role in the phase separation of the gephyrin/GlyR- $\beta$ LD complex. $20 \mu \mathrm{M}$ gephyrin or its variants were mixed with $40 \mu \mathrm{M}$ GlyR- $\beta$ LD. Data from three different batches of experiments were presented as means \pm SD. $f$ Schematic diagrams showing the regions of the gephyrin C-domain responsible for inhibiting E-domain-mediated phase separation with GlyR- $\beta$ LD. The C-domain is separated into three segments (Cl, Cll, and CIII). Two phase separation inhibitory regions ("Inhibitory Region 1" and "Inhibitory Region 2") are marked. The locations and sequences of two DLC1 binding regions ("DLC1 BR1" and "DLC1 BR2") are also indicated. The three previously identified phosphorylation sites (S268, S270, S305) are highlighted in red. See also Supplementary information, Fig. S8. 
The enrichments of the dimeric GlyR- $\beta$ LD in gephyrin puncta are significantly higher than the monomeric GlyR- $\beta$ L (Fig. 4a), presumably due to the increased valency of GlyR- $\beta$ LD. However, heterologous cells are not best suited for detailed mechanistic analysis of gephyrin-mediated phase separation, as gephyrin may undergo different levels of self-aggregation in cells. We resorted to purified recombinant full-length gephyrin (GPHN-FL) for studying its phase separation with GlyR- $\beta$ L. We predicted that GPHN-FL would have a higher capacity in forming phase-separated droplets upon binding to GlyR- $\beta$ LD as the trimerization G-domain could significantly increase the valency of the GPHN-FL/GlyR- $\beta$ LD complex. Surprisingly, GPHN-FL displayed a much more reduced capacity in forming phase-separated droplets with GlyR- $\beta$ LD (e.g., no phase separation occurred when both proteins were at concentrations as high as $20 \mu \mathrm{M}$; Fig. 4c; Supplementary information, Fig. S8a). When fixing GPHN-FL at $20 \mu \mathrm{M}$, increasing the concentration of GlyR- $\beta$ LD led to a progressive increase of phase separation of the mixtures (Fig. 4c, d; Supplementary information, Fig. S8a).

Consistent with earlier studies, ${ }^{34,42,45}$ GPHN-FL purified from $E$. coli formed multiple forms of oligomers. The prevailing fraction of GPHN-FL has a molecular mass higher than a dimer but lower than a trimer (Supplementary information, Fig. S8b), suggesting that GPHN-FL does not form a hexamer or lattice sheet-like structure in solution. GlyR- $\beta$ LD binds to GPHN-FL with a slightly increased affinity than to GPHN-E (Supplementary information, Fig. S8c). Thus, the reduced phase separation capacity of GPHN-FL was not due to the blocking of the interaction between GlyR- $\beta$ LD and GPHN-FL.

To figure out why GPHN-FL has a reduced phase separation capacity, we performed a domain mapping study of the protein. Under the assay condition with $20 \mu \mathrm{M}$ gephyrin (or its mutants) mixed with $40 \mu \mathrm{M}$ GlyR- $\beta L D$, truncating the G-domain (i.e., the gephyrin mutant containing $\mathrm{C}$ - and E-domains; "GPHN-CE") abolished its phase separation. In contrast, replacing the Cdomain of gephyrin with a 12-residue flexible linker (four repeats of the "GGS" tripeptide, denoted as GPHN-G E) showed a similar phase separation capacity as GPHN-E (Fig. 4e). This mapping experiment revealed that the $\mathrm{C}$-domain inhibits the $\mathrm{E}$-domainmediated phase separation of gephyrin with GlyR- $\beta$ LD. The fact that GPHN-FL had a higher capacity to phase separate with GlyR$\beta L D$ than GPHN-CE did (Fig. 4e) indicated the G-domain multimerization-promoted phase separation of GPHN-FL. Further detailed mapping revealed that two peptide fragments in the Cdomain (aa 259-274 in the middle of the C-domain and aa 303-318 immediately preceding the E-domain, Fig. 4f) inhibit the E-domain-mediated phase separation (see Supplementary information, Fig. S8d-f and additional text of the figure for the mapping details). The stretch of the positively charged residues immediately preceding the E-domain might interact with the negatively charged subdomain II of GPHN-E (Fig. 3d), blocking the second GlyR- $\beta$ L binding site on the domain (Supplementary information, Fig. S8g).

DLC1 promotes phase separation of gephyrin/GlyR- $\beta$ LD complex Dynein light chains (DLCs) interact with gephyrin C-domain and exhibit synaptic localization in cultured neurons. ${ }^{13}$ Thus, in addition to functioning as a possible cargo adaptor for dyneinmediated GlyRs transport, ${ }^{46,47}$ the homodimer property of DLCs may directly modulate the scaffolding capacity of gephyrin by promoting dimerization of its E-domain (i.e., increasing the valency of the E-domain). ${ }^{48,49}$

We mixed DLC1, GlyR- $\beta$ LD, and different versions of gephyrin proteins to test whether DLC1 might promote phase separation of gephyrin/GlyR- $\beta$ LD via increasing the valency of the complex. The addition of $20 \mu \mathrm{M}$ DLC1 to the mixture of $20 \mu \mathrm{M}$ GPHN-CE and 40 $\mu \mathrm{M}$ GlyR- $\beta$ LD led to phase separation of the GPHN-CE/GlyR- $\beta$ LD complex and co-enrichment of DLC1 in the condensed droplets
(Fig. 5a). The addition of $20 \mu \mathrm{M}$ DLC1 to the mixture of $20 \mu \mathrm{M}$ GPHN-FL and $40 \mu \mathrm{M}$ GlyR- $\beta$ LD significantly promoted the phase separation of the GPHN-FL/GlyR- $\beta$ LD complex (Fig. 5b, c). The DLC1 did not induce or promote phase separation of GPHN-CE or GPHN-FL in the absence of GlyR- $\beta$ LD (Fig. $5 c$; Supplementary information, Fig. S9a), reinforcing our earlier conclusion that the direct binding between gephyrin and GlyR- $\beta \mathrm{L}$ is the driving factor for the phase separation of the complex. The GPHN-C domain contains a strong (aa 203-212, $K_{\mathrm{D}} \sim 0.77 \mu \mathrm{M}$ ) and a weak (aa 246-258, $K_{\mathrm{D}} \sim 94 \mu \mathrm{M}$ ) DLC1 binding site (Fig. 4f, with mapping details presented in Supplementary information, Fig. S9). Removing one DLC1 binding site ("GPHN-CE_ $\triangle B R 1$ ") lowered and deleting both DLC1 binding sites ("GPHN-CE_ $\triangle \triangle B R^{\prime \prime}$ ) eliminated DLC1's capacity in promoting phase separation of the gephyrin/ GlyR- $\beta$ LD complex (Fig. 5d; Supplementary information, Fig. S9b, c). As the control, DLC1 had no impact on the phase separation of the GPHN-E/GlyR- $\beta$ LD complex (Supplementary information, Fig. S9d). In sum, the above results indicated that DLC1 binding to gephyrin promotes phase separation of the gephyrin/GlyR- $\beta$ LD complex most likely by increasing the valency of the complex interaction.

$G_{A B A_{A}} R$ via its a3 subunit forms phase-separated condensates with gephyrin

Several subunits ( $\alpha 1, \alpha 2, \alpha 3, \beta 2$, and $\beta 3$ ) of $G_{A B A}$ Rs have also been reported to interact with gephyrin, and the structures of GPHN-E/GABA ${ }_{A} R$ complexes showed that $G_{A B A}{ }_{A} R$ s share a similar binding mode with GlyR- $\beta$ L in binding to GPHN-E. ${ }^{50-53}$ Although the bindings between $G_{A B A_{A}} R s$ and gephyrin are considerably weaker than the interaction between GlyR and gephyrin, gephyrin is also essential for the clustering and transmissions of major $G_{A B A} R$ subtypes. ${ }^{54-57}$ To test whether $G_{A B A} R$ s might also phase separate with gephyrin, we generated dimeric GCN4-fused fulllength TM3-4 loops of $G A B A_{A} R$ a subunits (referred to as $G A B A_{A} R$ aLD) because each pentameric $G A B A_{A} R$ usually contains two a subunits (i.e., 2a:2 $2 \beta: 1 \gamma)^{58}$ (Figs. 1a, 6a). The sedimentation assay showed that only $G_{A B A}$ R-a3LD co-sedimented with GPHN-E at the protein concentration of $5 \mu \mathrm{M}$ or below (Fig. 6b; Supplementary information, Fig. S10c). GABA ${ }_{A}$ R-a1LD and-a2LD at elevated concentrations could also phase separate with GPHN-E (Supplementary information, Fig. S10a, b). The phase separation of the GPHN-E/GABA $A_{A} R-a 3 L D$ complex was concentration-dependent both in solution and on supported lipid bilayers (Fig. 6c, d; Supplementary information, Fig. S10c). The condensed phase formed by the GPHN-E/GABA ${ }_{A}$ R-a3LD complex is dynamic, as formed droplets could fuse with each other (Supplementary information, Fig. S10d). The phase separation of the GPHN-E/ $G_{A B A} R$-a3LD complex is also very sensitive to buffer salt concentrations (Fig. 6e; Supplementary information, Fig. S10e). Parallel to the observations in the GPHN-E/GlyR- $\beta$ LD complex, the phase separation of the GPHN-E/GABA ${ }_{A} R-a 3 L D$ complex requires the direct interaction between the two proteins as well as the multivalent properties of both proteins (Fig. 6f). Neutralization of the negatively charged surface of the GPHN-E subdomain II decreased its phase separation with $G A B A_{A} R-a 3 L D$, further suggesting that the GPHN-E/GABA ${ }_{A} R-a 3 L D$ and GPHN-E/GlyR$\beta L D$ complexes share a similar mechanism in phase separation (Supplementary information, Fig. S10f).

Mirroring the behavior of the gephyrin/GlyR- $\beta$ LD complex, coexpression of mCherry-GPHN with GFP-tagged GABA $A_{A} R$-a3LD led to perfect colocalization of the two proteins in bright puncta and consequently dramatic enrichment of $G A B A_{A} R$-a3LD in the gephyrin puncta (Fig. 6g). GFP-tagged $G_{A B A} R-a 3 L$ monomer (GFP-GABA $A_{A}$ R-a3LM) had a much lower capacity in phase

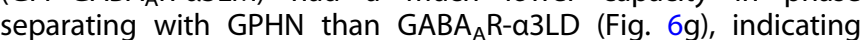
the role of multivalency in the complex phase separation. Again, like what was observed for GlyR- $\beta$ LD, GPHN-FL showed a lower

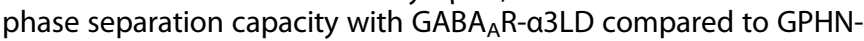



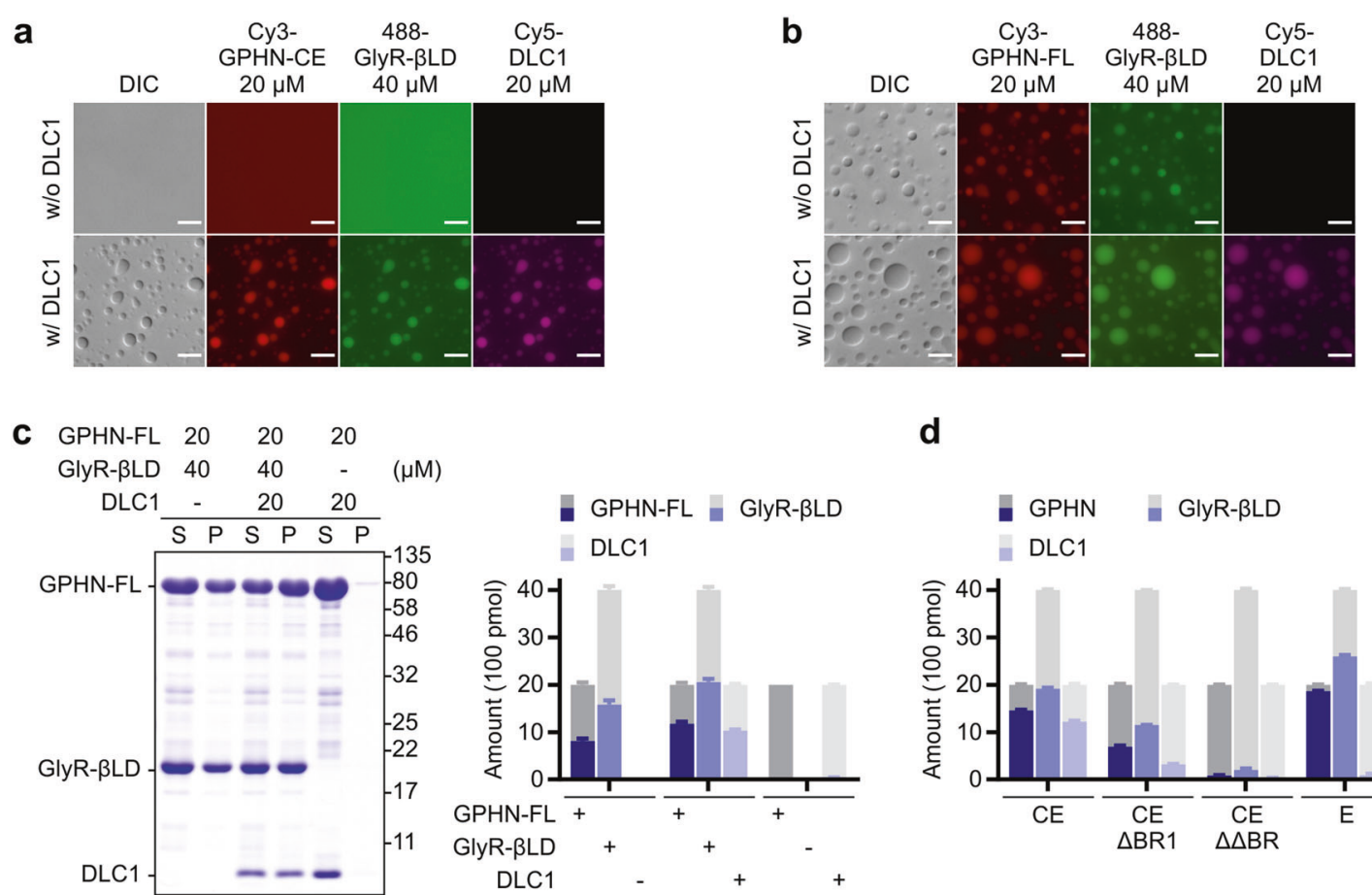

d

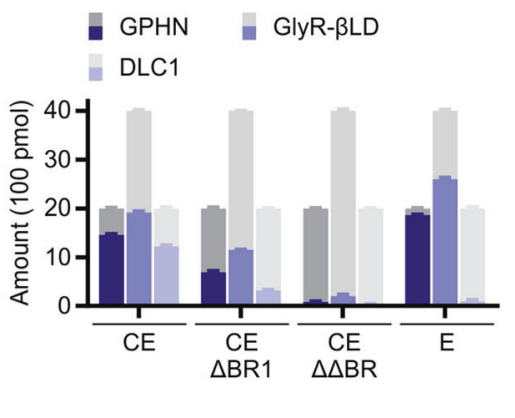

Fig. 5 DLC1 promotes phase separation of the gephyrin/GlyR- $\beta$ LD complex. $\mathbf{a}, \mathbf{b}$ DIC and fluorescence images showing that DLC1 was enriched in and promoted the phase separation of the Cy3-GPHN-CE/488-GlyR- $\beta$ LD complex (a) and the Cy3-GPHN-FL/488-GlyR- $\beta$ LD complex (b) at the indicated protein concentrations. Scale bars, $10 \mu \mathrm{m}$. c Representative SDS-PAGE of sedimentation experiments (left panel) and quantification of protein amounts in the supernatant ( $\mathrm{S}$, gray columns) and pellet ( $\mathrm{P}$, blue columns) (right panel) showing that DLC1 cosedimented and increased the condensate formation of the GPHN-FL/GlyR- $\beta$ LD complex. Proteins were mixed at indicated concentrations. Data from three different batches of experiments were presented as means \pm SD. $\mathbf{d}$ Quantification of protein amounts in the supernatant (S, gray columns) and pellet ( $P$, blue columns) in sedimentation experiments showing that deleting "DLC1 binding region 1" or "DLC1 binding regions $1 \& 2$ " weakened or even abolished DLC1-mediated enhancement of the phase separation of the GPHN-CE/GlyR- $\beta$ LD complex. See also Supplementary information, Fig. S9.

E (Figs. 6h, 4c). The decreased phase separation of GPHN-FL with $G A B A_{A} R-a 3 L D$ was also because of the C-domain-mediated autoinhibition of gephyrin (Supplementary information, Fig. S10g, h).

Phosphorylation of the $\mathrm{C}$-domain regulates the phase separation of gephyrin with $\mathrm{GABA}_{\mathrm{A}}$ Rs and GlyRs

In inhibitory synapses, activity-dependent phosphorylation of gephyrin are known to play key roles in synaptogenesis and synaptic plasticity.,10 Most of the phosphorylation sites are located within the C-domain, thus these phosphorylation events should not directly affect the interactions between gephyrin and $\mathrm{GABA}_{A} \mathrm{Rs} / \mathrm{GlyRs}$. The mechanisms underlying the dynamic regulation of $G_{A B A_{A} R / G l y R}$ clustering by gephyrin phosphorylation are poorly understood.

It is noted that S268 and S270, both in the first autoinhibitory region of the C-domain of gephyrin (Fig. 4f), could be phosphorylated by ERKs, GSK3 $\beta$, or CDK5. ${ }^{59-61}$ S305 is in the second autoinhibitory region $\mathrm{N}$-terminal to the $\mathrm{E}$-domain of gephyrin (Fig. 4f), and this residue has been reported to be a substrate of $\mathrm{Ca}^{2+}$ /calmodulin-dependent protein kinase $1 .^{62}$ Given that the gephyrin-mediated phase separations of $G A B A_{A} R s / G l y R s$ are highly sensitive to the charge-charge interactions between gephyrin and the receptors, it is likely that phosphorylation of the residues in the two autoinhibitory segments can regulate phase separation of the gephyrin/receptor complexes. Satisfyingly, the substitution of S305 of gephyrin with Glu enhanced its phase

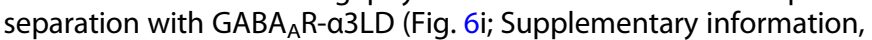
Fig. S10h), likely due to the neutralization of the positive charges in the autoinhibitory region and consequent weakening of the autoinhibition. Interestingly, substitutions of S268 or S270 with Glu were found to further weaken the phase separation of gephyrin

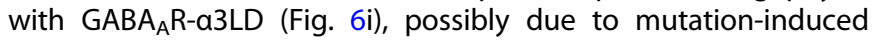
enhancement of autoinhibition by this segment. The above results point to exciting possibilities that phosphorylation of various residues in the C-domain can bidirectionally modulate gephyrinmediated clustering of $\mathrm{GABA}_{A} \mathrm{Rs} / \mathrm{Gly}$ Rs in inhibitory synapses via phase separation, though detailed additional studies are required in the future.

\section{DISCUSSION}

Phase separation has been implicated as a mechanism for forming various compartmentalized molecular assemblies in synapses including ePSDs, ${ }^{4,5}$ presynaptic active zones, ${ }^{63}$ and reserve pool synaptic vesicle clustering. ${ }^{64}$ In this study, we provide evidence to show that iPSD may also autonomously form via phase separation.

Due to the relatively low resolution of classical EM-based imaging, it has been rather elusive whether inhibitory synapses also contain highly condensed postsynaptic densities beneath plasma membranes. Recent high-resolution cryo-EM tomography studies revealed that inhibitory synapses also contain electrondense PSD assembly. ${ }^{6,28}$ In contrast to ePSDs, iPSDs are very thin and form a quite uniform layer with the thickness $\sim 5 \mathrm{~nm}$ right beneath the condensed $\mathrm{GABA}_{\mathrm{A}} \mathrm{Rs}$ on postsynaptic plasma membranes. ${ }^{28}$ Combining the results from the current study and the recent cryo-EM tomography study of the inhibitory synapse in situ, ${ }^{28}$ a compelling model depicting iPSD formation and iPSDmediated receptor clustering readily emerges (Fig. 7). Gephyrin, via its dimerization E-domain, binds to the cytoplasmic TM3-4 loops of selected subunits from $G A B A_{A}$ Rs or GlyRs. The multivalent nature of $\mathrm{GABA}_{A} \mathrm{Rs}$ or GlyRs can polymerize the gephyrin E- 
a

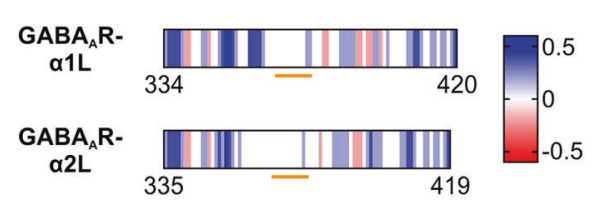

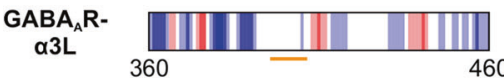

C

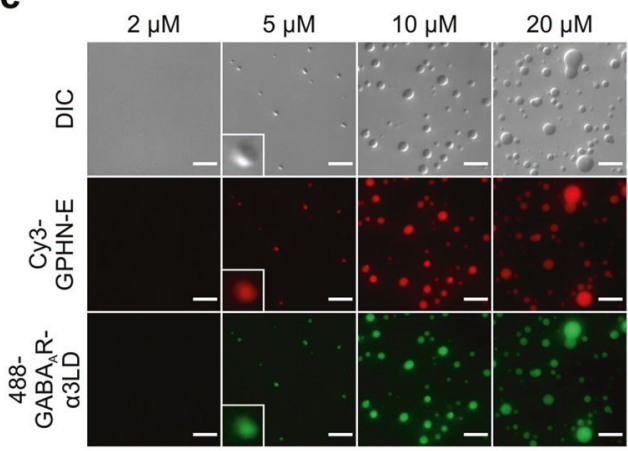

b $5 \mu \mathrm{M}$ GPHN-E $+5 \mu \mathrm{M} \alpha \mathrm{LD}$ GABA $_{A} R-\alpha 1 L D$ a2LD $\alpha 3 L D$

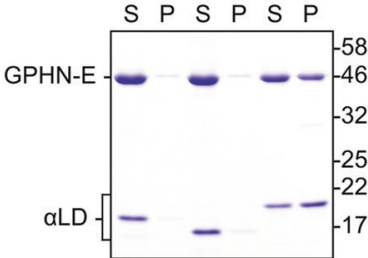

d 488-GABA $A_{A} R-\alpha 3 L D$ e

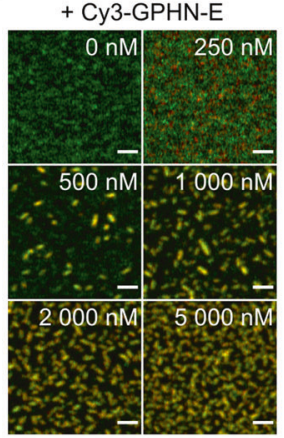

GPHN-E GABA $_{A}$
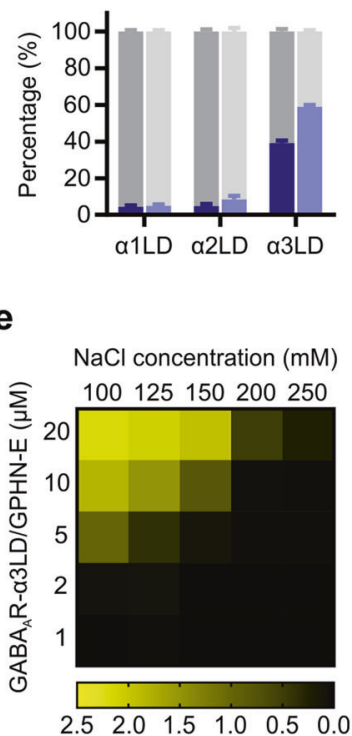

f

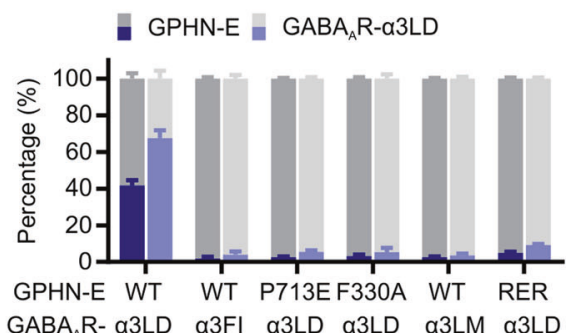

g - GFP-GABA ${ }_{A} R-\alpha 3 L M=G F P-G A B A_{A} R-\alpha 3 L D$ - mCherry-GPHN $=$ mCherry-GPHN
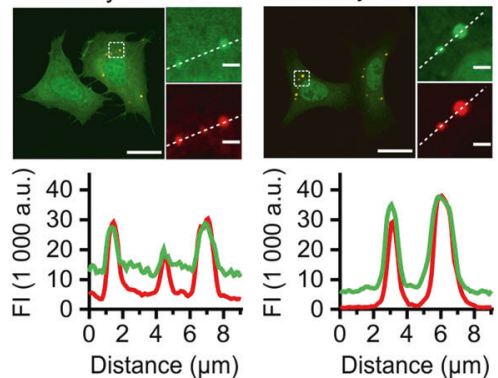

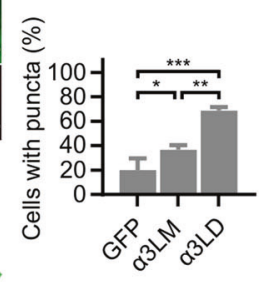

h

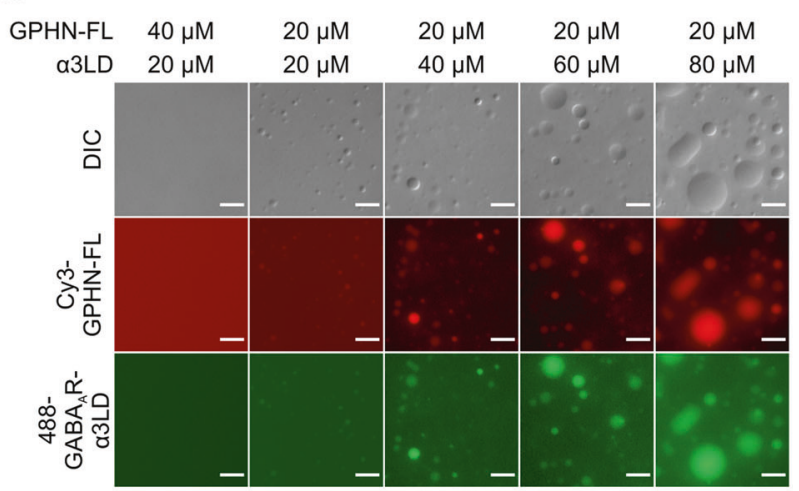

i $10 \mu \mathrm{M}$ GPHN-CE $+20 \mu \mathrm{M}$ GABA $A_{A}$ - $-\alpha 3 L D$ mM NaCl solution GPHN-CE WT S305E S268E S270E

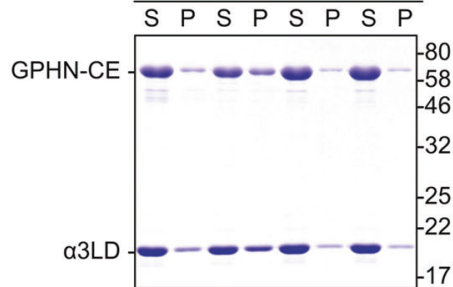

GPHN-CE $\quad$ GABA $_{A} R-\alpha 3 L D$

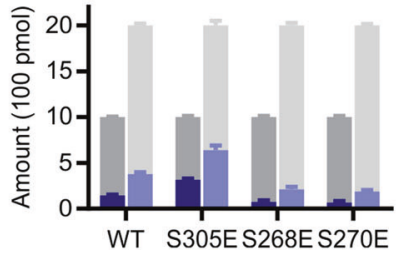

domain dimer into a sheet-like assembly via phase separation. In inhibitory synapses of spinal cord neurons, the stoichiometry of the GlyR $\beta$ subunit to gephyrin has been estimated to be $\sim 1: 1,65$ matching the sheet-like gephyrin/receptor organization. The width of a gephyrin E-domain dimer is $\sim 11 \mathrm{~nm}$ (Fig. 3d). This translates into the distance of the receptor binding sites between the two neighboring E-domain dimers to be $\sim 11 \mathrm{~nm}$ if the Edomain dimers are next to each other. Interestingly, the distance of the nearest neighbor distribution of $G A B A_{A} R s$ in inhibitory synapses measured by EM tomography is also centered at $\sim 11$ 
Fig. 6 Phase separation of the gephyrin/GABA $A_{A} R-a 3 L D$ complex. a Charge distributions of the cytoplasmic TM3-4 loops in the GABA $R$ $\alpha$ subunits. The positions of the core binding sequences for gephyrin are highlighted by orange lines. b Representative SDS-PAGE of sedimentation experiments (left panel) and quantification of protein distributions in the supernatant $(S$, gray columns) and pellet $(P$, blue columns) (right panel) showing the phase separation capacities of different $G_{A B A} R \alpha$ subunit TM3-4 loops. Data from three batches of experiments were presented as means \pm SD. c DIC and fluorescence images showing that mixtures of Cy3-GPHN-E and 488-GABA $R-\alpha 3 L D$ at the 1:1 molar ratio formed droplets at the indicated concentrations. The boxes show a $5 \times$ zoom-in analysis of a droplet at the $5 \mu \mathrm{M}$ of the protein concentration. Scale bars, $10 \mu \mathrm{m}$. d Fluorescence images showing that the clustering patterns of membrane-tethered $488-G A B A_{A} R-$ $\alpha 3 L D$ on lipid bilayers depended on the concentration of Cy3-GPHN-E added. Scale bars, $2 \mu \mathrm{m}$. e Phase diagram showing the phase separation of the GPHN-E/GABA ${ }_{A} \mathrm{R}-\alpha 3 \mathrm{LD}$ complex as functions of buffer $\mathrm{NaCl}$ concentration and protein concentrations. The degree of phase separation at each data point was derived from the turbidity assay as described in Fig. 2d. $\mathbf{f}$ Quantification of protein distributions in the supernatant ( $\mathrm{S}$, gray columns) and pellet (P, blue columns) showing the phase separation capacities of different GPHN-E mutants or GABA ${ }_{A} R-\alpha 3 L$ in different oligomerization states. Proteins were mixed at $5 \mu \mathrm{M}$ at the 1:1 ratio. Data from three different batches of experiments were presented as means $\pm S D$. g Left and middle panels: representative fluorescence images showing the co-expression of mCherry-GPHN with GFP-GABA ${ }_{A} R-$ $\alpha 3$ LM or with GFP-GABA $A_{A} R-\alpha 3 L D$ in HeLa cells. The region highlighted with a dashed box in each image is zoomed-in at right. Fluorescence intensity line-scanning plots showing the distribution profile of GFP-GABA ${ }_{A} R-\alpha 3 L M$ or GFP-GABA $A_{A} R-\alpha 3 L D$ with that of mCherry-GPHN as indicated by the dashed lines in each zoomed-in image. Scale bars, $20 \mu \mathrm{m}$. Scale bars for the zoomed-in images, $2 \mu \mathrm{m}$. Right panel: quantification of the percentage of cells with two proteins colocalized puncta over the total co-transfected cells. Data from three batches of cultures were presented as means $\pm \mathrm{SD}$. ${ }^{*} P<0.05 ;{ }^{* *} P<0.01 ;{ }^{* *} P<0.001$. $\mathbf{h}$ DIC and fluorescence images showing the phase separation of the Cy3-GPHN-FL/488-GABA ${ }_{A}$ R- $\alpha 3 L D$ mixtures at indicated molar ratios. Proteins were mixed at indicated concentrations in the imaging buffer containing $50 \mathrm{mM} \mathrm{NaCl}$. Scale bars, $10 \mu \mathrm{m}$. i Representative SDS-PAGE of sedimentation experiments (upper panel) and quantification of protein amounts in the supernatant ( $\mathrm{S}$, gray columns) and pellet ( $\mathrm{P}$, blue columns) (lower panel) showing that the phosphorylation of different sites in the gephyrin C-domain had different effects on the phase separation of the GPHN-CE/GABA ${ }_{A} R-\alpha 3 L D$ complex. $10 \mu M$ GPHN-CE and 20 $\mu \mathrm{M} G A B A_{A} R-\alpha 3 L D$ were mixed in the assay buffer containing $75 \mathrm{mM} \mathrm{NaCl}$. Data from three different batches of experiments were presented as means \pm SD.

$\mathrm{nm} .^{28}$ The thickness of the iPSD sheet also roughly matches the height of the E-domain dimer (i.e., $\sim 5 \mathrm{~nm}$; Fig. 3d). The G-domain of gephyrin is likely located beneath the iPSD sheet distal to the plasma membranes. Since the C-domain of gephyrin is largely unstructured, the trimerized G-domains do not show up in the EM tomograms. Nonetheless, the trimerization of the G-domain promotes the phase separation of the gephyrin/receptor complex by increasing the valency of the assembly.

Importantly, the phase separation of the gephyrin/GABA $R$ or GlyR complexes (and likely clustering of the receptors in inhibitory synapse) is regulated by the gephyrin C-domain (Fig. 7a), which inhibits the gephyrin/receptor phase separation via autoinhibition. Phosphorylation of specific residues in the C-domain may either strengthen or weaken the autoinhibition of gephyrin, providing a potential mechanism for bidirectional synaptic activity regulation by different kinases. Binding of proteins such as DLCs to the Cdomain of gephyrin can also regulate phase separation of the gephyrin/receptor complexes. In this case, DLCs promote phase separation also by increasing the valency of the gephyrin/receptor complexes. It will be interesting to study how other gephyrin Cdomain binders may regulate phase separation of the gephyrin/ receptor complexes.

Analogous to AMPARs in ePSD, recent studies have identified a number of transmembrane proteins in the complex with native $\mathrm{GABA}_{\mathrm{A}} \mathrm{Rs}$ including GARLH4/Lhfpl4 and Shisa7. These transmembrane proteins function as auxiliary subunits of $G A B A_{A} R s .{ }^{66,67}$ We tested whether these $G A B A_{A} R$ auxiliary subunits may interact with gephyrin and thus regulate the trafficking and clustering of the receptors in a way analogous to the TARP/PSD-95 interaction in ePSD. ${ }^{68}$ Unexpectedly, we could not detect any direct interaction between GARLH4 (or Shisa7) and gephyrin (data not shown), suggesting that $G A B A_{A} R$ auxiliary subunits may regulate the trafficking, clustering, and the function of $G A B A_{A} R s$ via binding to protein(s) other than gephyrin.

The assembly mechanism of the gephyrin/GABA $R$ or GlyR complex condensates also provides a possible explanation as to why iPSD does not grow thicker towards the cytoplasm. Unlike the multilayer structure formed by a group of major scaffold proteins such as PSD-95, SAPAP, Shank, and Homer in ePSD, ${ }^{3,5}$ gephyrin binding proteins in IPSD are neither highly concentrated in synapses like gephyrin nor are multi-domain scaffold proteins. Therefore, these gephyrin binding proteins may function as regulators rather than drivers of the gephyrin/GABA $R$ or GlyR complex condensates. Accordingly, these gephyrin binding proteins do not significantly promote the thickness of the iPSD assembly. The observation that the condensed assemblies of the gephyrin/GABA $R$ or GlyR complexes are highly sensitive to the salt concentration in the buffer may have certain implications. In addition to the possibility that the assembly of iPSD may be regulated by phosphorylation of gephyrin and receptors (Figs. 2a, $6 i)$, the sensitivity of the assemblies to the buffer condition may be a reason for not being able to biochemically purify iPSD from brain extracts (i.e., the iPSD assemblies could easily fall apart during sample handling). Certainly, the above speculations will need to be tested experimentally in the future. Nonetheless, the finding that the gephyrin/GABA $\mathrm{R}$ or GlyR complexes can form condensed assemblies via phase separation may open new research directions for understanding the structure and functions of inhibitory synapses.

\section{MATERIALS AND METHODS}

Plasmid constructs

The cDNA encoding full-length gephyrin from Rattus norvegicus (NM_022865) and DLC1 from Rattus norvegicus (NM_053319) were amplified from rat brain cDNA libraries. Various mutants of the proteins used in this study were generated by standard PCR-based methods and inserted into modified $\mathrm{pET}$ vectors with an $\mathrm{N}$ terminal $\mathrm{His}_{6}$ - or $\mathrm{TRX}-\mathrm{His}_{6}$-tag followed by an HRV-3C protease cutting site (pET.M3C/pET.32M3C) for protein purification. The genes encoding TM3-4 loop fragments of the GlyR $\beta$ subunit and its variants were synthesized based on the sequence of NM_010298. The genes encoding $G_{A B A} R$ a1-3 subunits (from Addgene plasmid \# 49168, \# 49169, and custom-synthesized based on the sequence of NM 000808, respectively) were each subcloned into pET.32M3C with an N-terminal $\mathrm{His}_{6}$-tag and a dimeric (or trimeric) GCN4 coiled-coil domain ${ }^{69}$ (referred to as $\mathrm{His}_{6}$-GlyR- $\beta$ LD or $\mathrm{His}_{6}-\mathrm{GABA} \mathrm{A}_{\mathrm{A}} \mathrm{R}-\mathrm{aLD}$ ). For heterologous expression, each GlyR $\beta$ or $G_{A B A_{A} R}$ fragment was cloned into modified pEGFP-C3 or pmCherry-C3 vectors. All constructs were verified by DNA sequencing.

Purification of recombinant proteins from $E$. coli Recombinant proteins were expressed in E. coli BL21 (DE3) cells (Agilent Technologies) in LB medium at $16^{\circ} \mathrm{C}$ for $16-20 \mathrm{~h}$ after IPTG induction. Protein purification was performed using $\mathrm{Ni}^{2+}$-NTA agarose affinity column followed by size-exclusion chromatography (SEC) with a column buffer containing $50 \mathrm{mM}$ Tris, $\mathrm{pH} 8.2$, 

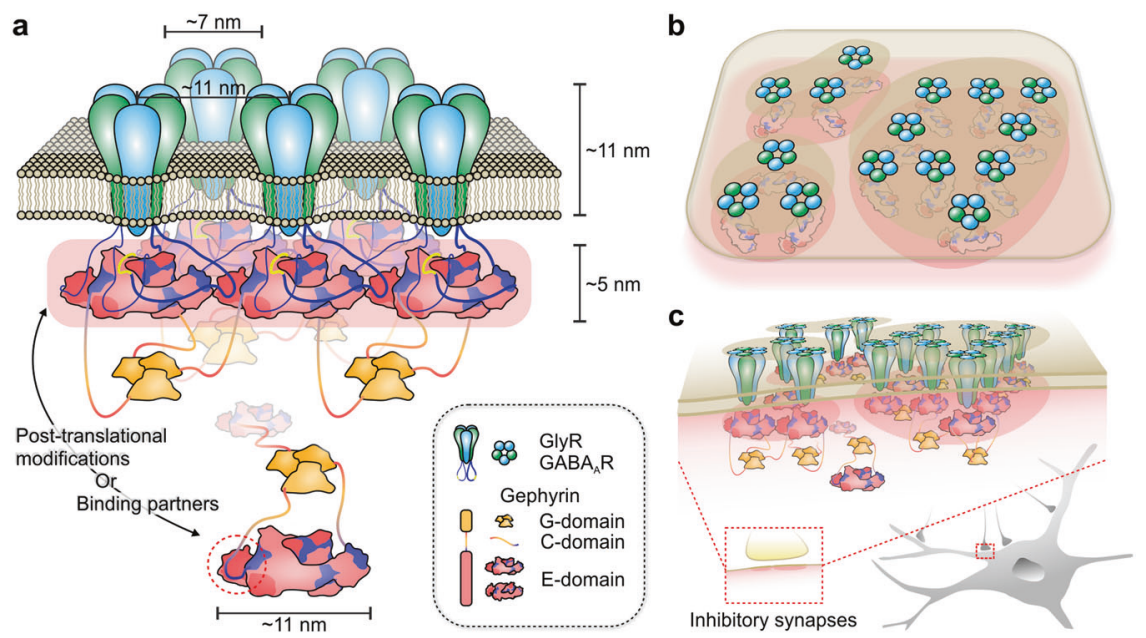

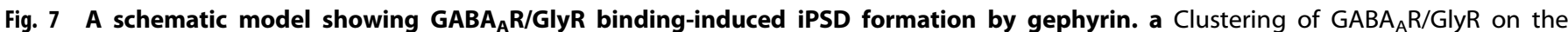
synaptic plasma membrane and formation of the gephyrin sheet beneath the synaptic membrane via receptor binding-induced phase separation. Note that the width of the gephyrin E-domain dimer is $\sim 11 \mathrm{~nm}$, and the distance between the two GABA $R$ receptors in iPSD is also $\sim 11 \mathrm{~nm} .^{28} \mathbf{b}$ A top-down view from the extracellular side of the synaptic membranes showing the phase separation-mediated $G A B A_{A} R /$ GlyR clustering and organization by the gephyrin condensates. c A schematic diagram showing progressive steps of zoomed-in views of an inhibitory synapse to show the formation of the iPSD sheet via phase separation.

100-500 mM NaCl, 1 mM EDTA, and 1 mM DTT. His $_{6}-/ T R X-$ His $_{6}$-tags were cleaved by HRV-3C protease at $4{ }^{\circ} \mathrm{C}$ overnight and removed by another step of SEC or ion-exchange chromatography. Purified proteins were finally exchanged into an assay buffer (used throughout the study unless otherwise noted) containing $25 \mathrm{mM}$ HEPES, pH 7.4, $100 \mathrm{mM} \mathrm{NaCl}$ and $1 \mathrm{mM}$ DTT.

\section{ITC}

ITC measurements were performed using a MicroCal VP-ITC calorimeter (Malvern). The experiment took place at $25^{\circ} \mathrm{C}$ in the assay buffer with $10-30 \mu \mathrm{M}$ of each indicated proteins in the sample cell titrated with its binder at 100-300 $\mu \mathrm{M}$. Typically, each titration point contained an injection of $10 \mu \mathrm{L}$ aliquot delivered in $20 \mathrm{~s}$ with a $120 \mathrm{~s}$ interval between successive injections. Highresolution isotherms were obtained by halving each titration to 5 $\mu \mathrm{L}$ without changing other conditions. Data were analyzed using the Origin 7 software.

Size-exclusion chromatography coupled with multiangle light scattering (SEC-MALS)

SEC-MALS assay was performed on an AKTA purifier system (GE Healthcare) coupled with a static light scattering detector (miniDawn, Wyatt) and a differential refractive index detector (Optilab, Wyatt). After ion-exchange chromatography, a homogeneous fraction of GPHN-FL was loaded to a Superose $610 /$ $300 \mathrm{GL}$ column in a column buffer composed of $25 \mathrm{mM}$ HEPES, $\mathrm{pH}$ 7.4, $100 \mathrm{mM} \mathrm{NaCl}$ and $1 \mathrm{mM}$ DTT. Data were analyzed using ASTRA6 (Wyatt).

Sedimentation and imaging assays of in vitro phase separation The concentrations of proteins in all assays were determined by NanoDrop 2000 spectrophotometer (Thermo Scientific) and precleared by high-speed centrifuging at $25^{\circ} \mathrm{C}$ before each phase separation assay.

For sedimentation assays, proteins were mixed at designated combinations and conditions in $100 \mu \mathrm{L}$ final volume. After $10 \mathrm{~min}$ incubation at room temperature, the mixtures were centrifuged at $16,873 \times g$ for $10 \mathrm{~min}$ at $25^{\circ} \mathrm{C}$. Right after centrifugation, the supernatant and pellet were separated. The pellet was brought to the same volume as the supernatant. Proteins recovered in supernatant and pellet were analyzed by the Bradford assays or SDS-PAGE with Coomassie Blue staining. The band intensities in
SDS-PAGE gels were quantified by ImageJ software. Relative and absolute amounts of proteins in supernatant and pellet were calculated based on the input amounts and relative band intensities. Data of three repeats were presented as means \pm SD.

DIC and fluorescence imaging assays were performed on a Nikon $\mathrm{Ni}-\mathrm{U}$ upright fluorescence microscope. Proteins were sparsely labeled with Alexa Fluor 488 NHS ester (ThermoFisher), Cy3 NHS ester (AAT Bioquest), or Cy5 NHS ester (AAT Bioquest) at the final labeling ratio of $2 \%$ (i.e., by mixing each labeled protein with unlabeled protein at the ratio of 2:98). The imaging buffer contains $50 \mathrm{mM}$ Tris, pH 7.4, $100 \mathrm{mM} \mathrm{NaCl}$, and $1 \mathrm{mM}$ TCEP. Images were captured after protein mixtures were injected into and settled onto the surface of homemade flow chambers ${ }^{4}$ or at the indicated time.

\section{Sample turbidity assay}

The solution turbidity was measured as absorption of each mixture at $350 \mathrm{~nm}$ using a $1 \mathrm{~cm}$ path-length cuvette at room temperature. The sample condition followed those used in the ITC experiments. Each titration was repeated three times.

Supported lipid bilayer preparation and 2D phase separation assay Supported lipid bilayers were prepared as described in the previous studies. ${ }^{5,63}$ Briefly, POPC (Avantilipids, Cat\# 850457), DGS$\mathrm{NTA}^{-\mathrm{Ni}^{2+}}$ (Avantilipids, Cat\# 790404), and PEG 5000 PE (Avantilipids, Cat\# 880230) were dissolved in chloroform. To generate multilamellar vesicles, mixtures of $98 \%$ POPC, $2 \%$ DGS-NTA-Ni ${ }^{2+}$, and $0.1 \%$ PEG 5000 PE were dried under a stream of nitrogen gas, resuspended and hydrated by PBS buffer with a final concentration of $0.5 \mathrm{mg} / \mathrm{mL}$. After overnight incubation at $4{ }^{\circ} \mathrm{C}$, the lipid solution was frozen and thawed repeatedly in combination with sonication to make it clear. The supernatant containing small unilamellar vesicles (SUVs) after centrifuging at $33,500 \times g$ for 45 min at $4{ }^{\circ} \mathrm{C}$ was collected for use.

Chambered cover glass (Lab-tek) was washed with Hellmanex II (Hëlma Analytics) overnight followed by immersed in $5 \mathrm{M} \mathrm{NaOH}$ for $1 \mathrm{~h}$ at $50^{\circ} \mathrm{C}$ and thoroughly rinsed with MilliQ water. Before use, the cover glass was equilibrated with the imaging buffer ( 50 $\mathrm{mM}$ Tris, pH 7.4, $100 \mathrm{mM} \mathrm{NaCl}, 1 \mathrm{mM}$ TCEP). SUVs $(75 \mu \mathrm{g})$ were coated on each glass for $1 \mathrm{~h}$ at $42{ }^{\circ} \mathrm{C}$ to generate supported lipid bilayers. Extra SUVs were washed out with the imaging buffer and the washing was repeated three times. The lipid bilayers were blocked by incubating the coated cover glass with the imaging 
buffer containing $1 \mathrm{mg} / \mathrm{mL}$ BSA for $30 \mathrm{~min}$ at room temperature. After blocking, Alexa Fluor 488-labeled $\mathrm{His}_{6}-\mathrm{GlyR}-\beta \mathrm{LD}$ or $\mathrm{His}_{6}{ }^{-}$ $\mathrm{GABA}_{\mathrm{A}} \mathrm{R}-\mathrm{a} 3 \mathrm{LD}$ dissolved in the imaging buffer containing $1 \mathrm{mg} / \mathrm{mL}$ BSA solution was incubated with lipid bilayers for $1 \mathrm{~h}$ at room temperature. Unbound proteins were washed away with the imaging buffer containing $1 \mathrm{mg} / \mathrm{mL}$ BSA solution. Different concentrations of test proteins were then added to induce phase separation on the lipid bilayers.

FRAP assay

FRAP experiments were carried out on a Zeiss LSM 880 confocal microscope at room temperature. To analyze condensates in solution, after $10 \mathrm{~min}$ settling down, a square region containing one droplet with the side length of $3.95 \mu \mathrm{m}$ was assayed. For the phase separation on lipid bilayers, a square region with a side length of $6.59 \mu \mathrm{m}$ was selected for FRAP analysis. Cy3 and 488 signals were bleached using $561 \mathrm{~nm}$ or $488 \mathrm{~nm}$ laser beam at $100 \%$ power, respectively. All experiments were completed within $30 \mathrm{~min}$ after initiation of the phase separations. For quantitative analysis, the average intensity of frames before photobleaching was normalized to $100 \%$, and the intensity right after the bleaching was set to $0 \%$. Data were analyzed using GraphPad Prism.

\section{Cell culture and imaging}

HeLa cells (from ATCC) were cultured in DMEM media supported by $10 \%$ fetal bovine serum maintained at $37{ }^{\circ} \mathrm{C}$ with $5 \% \mathrm{CO}_{2}$. The cells were transfected after $24 \mathrm{~h}$ plating at approximately $75 \%$ confluency using ViaFect transfection kit (Promega, Cat\# E4982). After incubating for another 20-24h, cells were fixed with $4 \%$ paraformaldehyde, 4\% sucrose in PBS. Images were captured on a Nikon Ni-U upright fluorescence microscope or a Zeiss LSM 880 confocal microscope. Quantitative data were derived from three independent batches of cultures in a blinded fashion. In each group, at least 500 fluorescence signal-positive cells were counted.

For the FRAP assay, $10 \mathrm{~h}$ after transfection, HeLa cells were incubated in a humidified chamber with $5 \% \mathrm{CO}_{2}$ at $37^{\circ} \mathrm{C}$. Images were captured on a Zeiss LSM 880 confocal microscope. A square region with a side length of $3.29 \mu \mathrm{m}$ was performed for FRAP. mCherry and GFP signals were bleached using $561 \mathrm{~nm}$ or $488 \mathrm{~nm}$ laser beam at $100 \%$ power, respectively. Images were taken in 5 min at the $2 \mathrm{~s} /$ frame and photobleaching was conducted after the third frame. For quantitative analysis, the average intensity of the first three frames before the bleach point was normalized to $100 \%$, and the intensity right after the bleaching was set to $0 \%$. Data were analyzed using GraphPad Prism.

For the lipid membrane staining by the lipophilic dye, $4 \mathrm{~h}$ after transfection, HeLa cells were incubated with DiD staining medium (DiD perchlorate, from ATT Bioquest dissolved at $1 \mathrm{mM}$ in DMSO and diluted to the final concentration of $1 \mu \mathrm{M}$ in DMEM) at $37^{\circ} \mathrm{C}$ for 20 $\min$. Cells were then washed three times each with $1 \mathrm{~mL}$ DMEM for $5 \mathrm{~min}$. Washed cells were cultured for another $6 \mathrm{~h}$ before fixing with $4 \%$ paraformaldehyde, $4 \%$ sucrose in PBS for imaging.

Quantification and statistical analysis

Statistical parameters including the definitions and exact values of $\mathrm{n}$ (e.g., number of experiments, number of droplets, etc), distributions, and deviations are reported in the figures and corresponding figure legends. Data on in vitro phase separation sedimentation assay and HeLa cell quantification were expressed as means \pm SD. ns, not significant; ${ }^{*} P<0.05 ;{ }^{*} P<0.01 ;{ }^{* *} P<$ $0.001 ;{ }^{* * *} P<0.0001$ using one-way ANOVA with Tukey's multiple comparison test. Statistical analysis was performed in GraphPad Prism.

\section{ACKNOWLEDGEMENTS}

This work was supported by a grant from the Minister of Science and Technology of China (2019YFA0508402), a grant from Human Frontier Science Program (RGP0020/
2019), and grants from Research Grant Council of Hong Kong (AoE-M09-12 and C6004-17G) to M.Z. M.Z. is a Kerry Holdings Professor of Science, a Croucher Foundation Senior Fellow, and a Senior Fellow of IAS at HKUST.

\section{AUTHOR CONTRIBUTIONS}

G.B., Y.W. performed experiments, G.B. and M.Z. analyzed the data, G.B. and M.Z. designed research, G.B. and M.Z. drafted and all authors commented on the paper, M.Z. coordinated the project.

\section{ADDITIONAL INFORMATION}

Supplementary information accompanies this paper at https://doi.org/10.1038/ s41422-020-00433-1.

Competing interests: The authors declare no competing interests.

\section{REFERENCES}

1. Biederer, T., Kaeser, P. S. \& Blanpied, T. A. Transcellular nanoalignment of synaptic function. Neuron 96, 680-696 (2017).

2. Sudhof, T. C. Towards an understanding of synapse formation. Neuron 100, 276-293 (2018)

3. Chen, X., Wu, X., Wu, H. \& Zhang, M. Phase separation at the synapse. Nat. Neurosci. 23, 301-310 (2020).

4. Zeng, M. et al. Phase transition in postsynaptic densities underlies formation of synaptic complexes and synaptic plasticity. Cell 166, 1163-1175 (2016).

5. Zeng, M. et al. Reconstituted postsynaptic density as a molecular platform for understanding synapse formation and plasticity. Cell 174, 1172-1187 (2018).

6. Tao, C. L. et al. Differentiation and characterization of excitatory and inhibitory synapses by cryo-electron tomography and correlative microscopy. J. Neurosci. 38, 1493-1510 (2018).

7. Pennacchietti, F. et al. Nanoscale molecular reorganization of the inhibitory postsynaptic density is a determinant of GABAergic synaptic potentiation. J. Neurosci. 37, 1747-1756 (2017).

8. Crosby, K. C. et al. Nanoscale subsynaptic domains underlie the organization of the inhibitory synapse. Cell Rep. 26, 3284-3297 (2019).

9. Tyagarajan, S. K. \& Fritschy, J. M. Gephyrin: a master regulator of neuronal function? Nat. Rev. Neurosci. 15, 141-156 (2014).

10. Alvarez, F. J. Gephyrin and the regulation of synaptic strength and dynamics at glycinergic inhibitory synapses. Brain Res. Bull. 129, 50-65 (2017).

11. Kirsch, J. et al. The 93-kDa glycine receptor-associated protein binds to tubulin. J. Biol. Chem. 266, 22242-22245 (1991).

12. Prior, P. et al. Primary structure and alternative splice variants of gephyrin, a putative glycine receptor-tubulin linker protein. Neuron 8, 1161-1170 (1992).

13. Fuhrmann, J. C. et al. Gephyrin interacts with Dynein light chains 1 and 2 , components of motor protein complexes. J. Neurosci. 22, 5393-5402 (2002).

14. Zhu, J., Shang, Y. \& Zhang, M. Mechanistic basis of MAGUK-organized complexes in synaptic development and signalling. Nat. Rev. Neurosci. 17, 209-223 (2016).

15. Pfeiffer, F., Graham, D. \& Betz, H. Purification by affinity chromatography of the glycine receptor of rat spinal cord. J. Biol. Chem. 257, 9389-9393 (1982).

16. Kasaragod, V. B. \& Schindelin, H. Structure-function relationships of glycine and GABAA receptors and their interplay with the scaffolding protein gephyrin. Front. Mol. Neurosci. 11, 317 (2018).

17. Xiang, S., Nichols, J., Rajagopalan, K. V. \& Schindelin, H. The crystal structure of Escherichia coli MoeA and its relationship to the multifunctional protein gephyrin. Structure 9, 299-310 (2001).

18. Sander, B. et al. Structural characterization of gephyrin by AFM and SAXS reveals a mixture of compact and extended states. Acta Crystallogr. D. Biol. Crystallogr. 69, 2050-2060 (2013).

19. Fritschy, J. M., Harvey, R. J. \& Schwarz, G. Gephyrin: where do we stand, where do we go? Trends Neurosci. 31, 257-264 (2008).

20. Fischer, F. et al. Reduced synaptic clustering of GABA and glycine receptors in the retina of the gephyrin null mutant mouse. J. Comp. Neurol. 427, 634-648 (2000).

21. Feng, G. et al. Dual requirement for gephyrin in glycine receptor clustering and molybdoenzyme activity. Science 282, 1321-1324 (1998).

22. Lionel, A. C. et al. Rare exonic deletions implicate the synaptic organizer Gephyrin (GPHN) in risk for autism, schizophrenia and seizures. Hum. Mol. Genet. 22, 2055-2066 (2013).

23. Groeneweg, F. L., Trattnig, C., Kuhse, J., Nawrotzki, R. A. \& Kirsch, J. Gephyrin: a key regulatory protein of inhibitory synapses and beyond. Histochem. Cell Biol. 150, 489-508 (2018). 
24. Banani, S. F., Lee, H. O., Hyman, A. A. \& Rosen, M. K. Biomolecular condensates: organizers of cellular biochemistry. Nat. Rev. Mol. Cell Biol. 18, 285-298 (2017).

25. Shin, Y. \& Brangwynne, C. P. Liquid phase condensation in cell physiology and disease. Science 357, eaaf4382 (2017).

26. Chen, X., Wu, X., Wu, H. \& Zhang, M. Phase separation at the synapse. Nat Neurosci 23, 301-310 (2020)

27. Wu, X., Cai, Q., Feng, Z. \& Zhang, M. Liquid-Liquid Phase Separation in Neuronal Development and Synaptic Signaling. Dev Cell 55, 18-29 (2020).

28. Liu, Y.-T. et al. Mesophasic organization of GABAA receptors in hippocampal inhibitory synapse. Preprint at bioRxiv https://doi.org/10.1101/2020.01.06.895425 (2020).

29. Lynch, J. W. Molecular structure and function of the glycine receptor chloride channel. Physiol. Rev. 84, 1051-1095 (2004).

30. Durisic, N. et al. Stoichiometry of the human glycine receptor revealed by direct subunit counting. J. Neurosci. 32, 12915-12920 (2012).

31. Lynch, J. W. Native glycine receptor subtypes and their physiological roles. Neuropharmacology 56, 303-309 (2009).

32. Dutertre, S., Becker, C. M. \& Betz, H. Inhibitory glycine receptors: an update. J. Biol. Chem. 287, 40216-40223 (2012).

33. Meyer, G., Kirsch, J., Betz, H. \& Langosch, D. Identification of a gephyrin binding motif on the glycine receptor beta subunit. Neuron 15, 563-572 (1995).

34. Sola, M. et al. Structural basis of dynamic glycine receptor clustering by gephyrin. $E M B O$ J. 23, 2510-2519 (2004).

35. Kim, E. Y. et al. Deciphering the structural framework of glycine receptor anchoring by gephyrin. EMBO J. 25, 1385-1395 (2006).

36. Grünewald, N. et al. Sequences flanking the Gephyrin-binding site of GlyR $\beta$ tune receptor stabilization at synapses. ENEURO. 5, 0042-17 (2018).

37. Meier, J., Vannier, C., Serge, A., Triller, A. \& Choquet, D. Fast and reversible trapping of surface glycine receptors by gephyrin. Nat. Neurosci. 4, 253-260 (2001).

38. Specht, C. G. et al. Regulation of glycine receptor diffusion properties and gephyrin interactions by protein kinase C. EMBO J. 30, 3842-3853 (2011).

39. Li, P. et al. Phase transitions in the assembly of multivalent signalling proteins. Nature 483, 336-340 (2012).

40. Grudzinska, J. et al. The beta subunit determines the ligand binding properties of synaptic glycine receptors. Neuron 45, 727-739 (2005).

41. Yang, Z., Taran, E., Webb, T. I. \& Lynch, J. W. Stoichiometry and subunit arrangement of alpha1beta glycine receptors as determined by atomic force microscopy. Biochemistry 51, 5229-5231 (2012).

42. Saiyed, T. et al. Molecular basis of gephyrin clustering at inhibitory synapses: role of G- and E-domain interactions. J. Biol. Chem. 282, 5625-5632 (2007).

43. Kneussel, M., Hermann, A., Kirsch, J. \& Betz, H. Hydrophobic interactions mediate binding of the glycine receptor beta-subunit to gephyrin. J. Neurochem. 72, 1323-1326 (1999).

44. Dejanovic, B. et al. Simultaneous impairment of neuronal and metabolic function of mutated gephyrin in a patient with epileptic encephalopathy. EMBO Mol. Med. 7, 1580-1594 (2015).

45. Herweg, J. \& Schwarz, G. Splice-specific glycine receptor binding, folding, and phosphorylation of the scaffolding protein gephyrin. J. Biol. Chem. 287, 12645-12656 (2012).

46. Hanus, C., Vannier, C. \& Triller, A. Intracellular association of glycine receptor with gephyrin increases its plasma membrane accumulation rate. J. Neurosci. 24, 1119-1128 (2004).

47. Maas, C. et al. Neuronal cotransport of glycine receptor and the scaffold protein gephyrin. J. Cell Biol. 172, 441-451 (2006).
48. Lo, K. W., Naisbitt, S., Fan, J. S., Sheng, M. \& Zhang, M. The 8-kDa dynein light chain binds to its targets via a conserved (K/R)XTQT motif. J. Biol. Chem. 276 14059-14066 (2001).

49. Fan, J., Zhang, Q., Tochio, H., Li, M. \& Zhang, M. Structural basis of diverse sequence-dependent target recognition by the $8 \mathrm{kDa}$ dynein light chain. J. Mol. Biol. 306, 97-108 (2001).

50. Tretter, V. et al. The clustering of $\operatorname{GABA}(\mathrm{A})$ receptor subtypes at inhibitory synapses is facilitated via the direct binding of receptor alpha 2 subunits to gephyrin. J. Neurosci. 28, 1356-1365 (2008).

51. Mukherjee, J. et al. The residence time of $G A B A(A) R s$ at inhibitory synapses is determined by direct binding of the receptor alpha1 subunit to gephyrin. $J$. Neurosci. 31, 14677-14687 (2011).

52. Tretter, V. et al. Molecular basis of the gamma-aminobutyric acid A receptor alpha3 subunit interaction with the clustering protein gephyrin. J. Biol. Chem. 286, 37702-37711 (2011).

53. Kowalczyk, S. et al. Direct binding of GABAA receptor beta2 and beta3 subunits to gephyrin. Eur. J. Neurosci. 37, 544-554 (2013).

54. Jacob, T. C. et al. Gephyrin regulates the cell surface dynamics of synaptic GABAA receptors. J. Neurosci. 25, 10469-10478 (2005).

55. $\mathrm{Yu}, \mathrm{W}$. et al. Gephyrin clustering is required for the stability of GABAergic synapses. Mol. Cell. Neurosci. 36, 484-500 (2007).

56. Choii, G. \& Ko, J. Gephyrin: a central GABAergic synapse organizer. Exp. Mol. Med. 47, e158 (2015).

57. Chiu, C. Q., Barberis, A. \& Higley, M. J. Preserving the balance: diverse forms of long-term GABAergic synaptic plasticity. Nat. Rev. Neurosci. 20, 272-281 (2019).

58. Tretter, V., Ehya, N., Fuchs, K. \& Sieghart, W. Stoichiometry and assembly of a recombinant GABAA receptor subtype. J. Neurosci. 17, 2728-2737 (1997).

59. Tyagarajan, S. K. et al. Regulation of GABAergic synapse formation and plasticity by GSK3beta-dependent phosphorylation of gephyrin. Proc. Natl. Acad. Sci. U S A. 108, 379-384 (2011).

60. Tyagarajan, S. K. et al. Extracellular signal-regulated kinase and glycogen synthase kinase 3beta regulate gephyrin postsynaptic aggregation and GABAergic synaptic function in a calpain-dependent mechanism. J. Biol. Chem. 288 9634-9647 (2013).

61. Kalbouneh, H., Schlicksupp, A., Kirsch, J. \& Kuhse, J. Cyclin-dependent kinase 5 is involved in the phosphorylation of gephyrin and clustering of GABAA receptors at inhibitory synapses of hippocampal neurons. PLoS One 9, e104256 (2014).

62. Flores, C. E. et al. Activity-dependent inhibitory synapse remodeling through gephyrin phosphorylation. Proc. Natl. Acad. Sci. U S A. 112, E65-E72 (2015).

63. $\mathrm{Wu}, \mathrm{X}$. et al. RIM and RIM-BP form presynaptic active-zone-like condensates via phase separation. Mol. Cell 73, 971-984 (2019).

64. Milovanovic, D., Wu, Y., Bian, X. \& De Camilli, P. A liquid phase of synapsin and lipid vesicles. Science 361, 604-607 (2018).

65. Specht, C. G. et al. Quantitative nanoscopy of inhibitory synapses: counting gephyrin molecules and receptor binding sites. Neuron 79, 308-321 (2013).

66. Yamasaki, T., Hoyos-Ramirez, E., Martenson, J. S., Morimoto-Tomita, M. \& Tomita S. GARLH family proteins stabilize GABAA receptors at synapses. Neuron 93, 1138-1152 (2017).

67. Han, W. Y. et al. Shisa7 isa GABA(A) receptor auxiliary subunit controlling benzodiazepine actions. Science 366, 246 (2019).

68. Zeng, M. et al. Phase separation-mediated TARP/MAGUK complex condensation and AMPA receptor synaptic transmission. Neuron 104, 529-543 (2019).

69. Harbury, P. B., Zhang, T., Kim, P. S. \& Alber, T. A switch between two-, three-, and four-stranded coiled coils in GCN4 leucine zipper mutants. Science 262, 1401-1407 (1993). 